QUARTERLY OF APPLIED MATHEMATICS

VOLUME LXXI, NUMBER 1

MARCH 2013, PAGES 89-117

S 0033-569X(2012)01275-7

Article electronically published on October 12, 2012

\title{
ASYMPTOTIC OF THE VELOCITY OF A DILUTE SUSPENSION OF DROPLETS WITH INTERFACIAL TENSION
}

\author{
BY \\ ERIC BONNETIER (Laboratoire Jean Kuntzmann, Université de Joseph Fourier \& CNRS, 38041 \\ Grenoble Cedex 9, France), \\ DAVID MANCEAU (Laboratoire de Mathématiques appliquées du Havre, Université du Havre, 25 \\ rue Philippe Lebon, BP 540, 76058 Le Havre cedex, France), \\ AND \\ FAOUZI TRIKI (Laboratoire Jean Kuntzmann, Université de Joseph Fourier $\&$ CNRS, 38041 \\ Grenoble Cedex 9, France)
}

\begin{abstract}
In this paper we derive the asymptotic expansion of the velocity field of a small deformable droplet immersed in an incompressible Newtonian fluid. Using an appropriate physical scaling of the surface tension with respect to the droplet volume, we show that the first order of the asymptotic can be expressed in terms of the velocity field in the absence of the droplet and a new kind of moment tensor, called the curvature moment tensor. Our asymptotic formula extends those already derived for rigid droplets and aimed to obtain simplified macroscale properties of a dilute suspension composed of identical droplets dispersed in an incompressible Newtonian fluid from knowledge of its microscopic properties. We finally determine explicitly the curvature moment tensor for ellipses and ellipsoids.
\end{abstract}

1. Introduction. Suspension of droplets has been a subject of active research for many years. A large body of work has been dedicated to the derivation of effective models of fluid carrying droplets, with famous contributions by A. Einstein [16, G.I. Taylor [25], G.K. Batchelor [11] among others.

In this work, we consider a dilute suspension of deformable droplets in a matrix fluid. We assume the droplet and matrix fluids to be Newtonian and neglect inertia and body forces. The evolution of droplet shape only depends on the viscosity difference between the fluids and on surface tension. The system is thus modeled as a stationary Stokes

Received March 30, 2011.

2010 Mathematics Subject Classification. Primary 35R30.

Key words and phrases. Stokes system, asymptotic expansion, surface tension, viscous moment tensor, droplets.

E-mail address: eric.bonnetier@imag.fr

E-mail address: david.manceau@univ-lehavre.fr

E-mail address: faouzi.triki@imag.fr

(C)2012 Brown University 
flow, in an open bounded domain $\Omega \subset \mathbb{R}^{n}$ that contains inhomogeneities (the droplets) of small size, say $\epsilon$. We further assume that the droplets are at a distance $\gg \varepsilon$ away from each other, and are also away from the boundary, to be able to neglect droplet interactions and their interaction with the boundary $\partial \Omega$.

We are interested in deriving an asymptotic expansion of the velocity $u_{\varepsilon}$ as the droplet diameter $\varepsilon$ tends to 0 . Our goal is to obtain in this manner an approximate model for the flow that only requires the resolution of a constant coefficient PDE to obtain the velocity $u_{0}$ of the homogeneous matrix fluid, and the computation of correction terms that depend on the viscosity ratio, and on the positions, the surface tensions, and the geometries of the droplets. Asymptotics of the fields in a smooth medium perturbed by inhomogeneities of small size have been derived in several contexts; see for example [5, 10, 12, 14]. The typical result establishes that the fields $u_{\varepsilon}$, in the presence of inhomogeneities centered at points $z_{j}$, and $u_{0}$, without inhomogeneities, are related by

$$
\left(u_{\varepsilon}-u_{0}\right)(x)=\varepsilon^{n} \sum_{j} M_{j} \nabla u_{0}\left(z_{j}\right) \cdot \nabla N\left(z_{j}, x\right)+o\left(\varepsilon^{n}\right),
$$

assuming that $x$ lies away from the centers $z_{j}$. Here, $n$ is the dimension of the ambient space, $N$ denotes a Neumann (or Green) function, and $M_{j}$ is the polarization matrix that encodes information on the material coefficient contrast and on the geometry of the $j$ th inhomogeneity. In other words, at the point $x$ the inhomogeneity is perceived like a dipole with moment $M_{j} \nabla u_{0}\left(z_{j}\right)$. The reader can find numerous examples of $M_{j}$ as well as its intrinsic properties in [3, 4, 6, 8, 9.

Our work is a continuation of the work of Ammari, Garapon, Kang and Lee [3, 4] on stationary Stokes flows. They considered droplets free of surface tension, in a homogeneous matrix fluid, as the limiting case of an elastic composite that contains small inclusions, as the coefficient $\lambda \rightarrow \infty$. They established an asymptotic of the form (11) for the velocity field and showed that the polarization tensor could be obtained as the limit when the Lamé coefficient $\lambda \rightarrow \infty$ of the corresponding polarization tensor for elasticity. Since one can also think of the polarization tensor as the first-order correction of the homogenized coefficients of a composite in the dilute limit [20, 23, they also recovered Einstein's expression for the effective viscosity of a suspension of rigid spherical particles.

In the present work, we incorporate surface tension. Since the curvature of the boundaries of the droplets scales like $\varepsilon^{-1}$, the surface tension has to be scaled appropriately with $\varepsilon$, so that the associated surface energy remains bounded. This is in agreement with the work of Tolman [27] who gave experimental evidence of how surface tension scales with the droplets' diameter. We show that an asymptotic expansion of the form (1) holds for the velocity. However, in addition to a viscous moment tensor, in the terminology of [4], the first-order term contains a contribution due to surface tension. We characterize this 'curvature moment tensor' and compute it explicitly when the inclusions are ellipses or ellipsoids.

The study of ellipsoidal droplets has been an active topic of work in recent years 22, 17. since ellipsoids may be good approximations to nearly spherical droplets (when surface tension is high) or to extended rod-like structures (when the viscosity of the droplets is small compared to the viscosity of the matrix). In these studies, a droplet 
is described by a morphology tensor $G$ such that $G_{i j} x_{i} x_{j}=1$ on the surface. An ODE describes the evolution of $G$, in terms of averaged expressions that resemble our viscosity or curvature moment tensor (see in particular [17, 28]) but for which only approximate expressions are derived.

The paper is organized as follows: In section 2, we describe our model for a droplet in a stationary Stokes flow and we state our main result: the structure of the first-order correction terms in the asymptotic expansion of the velocity. Section 3 contains the proof of the asymptotic formula. The next section is devoted to the explicit determination of the curvature moment tensors for ellipses and ellipsoids. Finally, an appendix recalls results on integration by parts on surfaces and on solvability of the Stokes problem in the whole space.

Notation.

- $I_{n}$ denotes the unit matrix in $\mathbb{R}^{n \times n}$, and $\mathbf{I}$ denotes the identity fourth-order tensor;

- $\mathbb{R}_{s}^{n \times n}$ denotes the set of symmetric $n \times n$ matrices;

- The scalar product on $\mathbb{R}^{n \times n}$ is defined by $A: B:=\operatorname{tr}\left(A^{t} B\right)$;

- $L_{0}^{2}(\Omega)$ denotes the space of the functions of $L^{2}(\Omega)$ which differ by a constant;

- The strain tensor of a field $u: \mathbb{R}^{n} \longrightarrow \mathbb{R}^{n}$ is

$$
e(u)=\frac{1}{2}\left(\nabla u+\nabla u^{T}\right)
$$

- The divergence of a matrix-valued function $A$ is denoted $\operatorname{Div}(A)$, while the divergence of a vector-valued function $u$ is $\operatorname{denoted} \operatorname{div}(u)$;

- Throughout the text, $c$ denotes a generic constant.

2. Statement of the main result. We consider a Stokes flow with viscosity $\mu_{0}>0$, in a bounded $C^{2}$-domain $\Omega$ in $\mathbb{R}^{n}(n=2,3)$. For simplicity, we assume that $\Omega$ contains a single droplet $\omega_{\varepsilon}=z_{0}+\varepsilon \omega$ of size $\varepsilon>0$, centered at a point $z_{0} \in \Omega$ with $\operatorname{dist}\left(z_{0}, \partial \Omega\right)>0$. The $C^{2}$-domain $\omega$ contains the origin and represents the rescaled shape of the droplet. We assume that $\omega_{\varepsilon}$ contains a Stokes fluid with viscosity $\mu_{1} \neq \mu_{0}$. We set

$$
\mu_{\varepsilon}(x):= \begin{cases}\mu_{0} & \text { if } x \in \Omega \backslash \bar{\omega}_{\varepsilon}, \\ \mu_{1} & \text { if } x \in \omega_{\varepsilon} .\end{cases}
$$

We want to compare the velocity $u_{\varepsilon}$ of the flow to the velocity $u$ of a reference flow in $\Omega$ when no droplet is present and the fluid is homogeneous with viscosity $\mu_{0}$. Let $g \in$ $C^{\infty}(\partial \Omega)^{n}$ such that

$$
\int_{\partial \Omega} g \cdot \nu_{x} d \sigma_{x}=0
$$

where $\nu_{x}$ denotes the outward unit normal to both $\partial \Omega$ and $\partial \omega_{\varepsilon}$. In view of (3), there exists a unique solution $(u, p) \in H^{1}(\Omega)^{n} \times L_{0}^{2}(\Omega)$ to the reference Stokes problem (see e.g. [26])

$$
\left\{\begin{aligned}
-\operatorname{Div}\left(2 \mu_{0} e(u)\right)+\nabla p & =0 & & \text { in } \Omega, \\
\operatorname{div}(u) & =0 & & \text { in } \Omega, \\
u & =g & & \text { on } \partial \Omega .
\end{aligned}\right.
$$


Classical elliptic regularity results (see [13] for $n=3$, and 26] for $n=2$ ) state that $u$ is $C^{\infty}$ in the interior of $\Omega$. We denote by $\kappa_{\varepsilon}$ the mean curvature on $\partial \omega_{\varepsilon}$. Note that since $\partial \omega_{\varepsilon}$ is $C^{2}, \kappa_{\varepsilon} \in L^{\infty}\left(\partial \omega_{\varepsilon}\right)$. Moreover,

$$
\kappa(y)=\varepsilon \kappa_{\varepsilon}\left(\varepsilon y+z_{0}\right), \quad \forall y \in \partial \omega,
$$

is the mean curvature on $\partial \omega$.

We assume that the contact of the fluids on $\partial \omega_{\varepsilon}$ creates a force of surface tension $\lambda_{\varepsilon} \kappa_{\varepsilon} \nu_{x}$. We consider Tolman's model [27, which proposes a scaling of the surface tension $\lambda_{\varepsilon}$ of the form

$$
\lambda_{\varepsilon}=\lambda \frac{\varepsilon}{\delta}=\tilde{\lambda} \varepsilon
$$

where $\delta$ is the constant Tolman length. This scaling is consistent with the scaling of the curvature, and we will see that this leads to a contribution at first order in the asymptotic expansion of the velocity, due to surface tension. If $\nu_{y}$ denotes the outward unit normal to $\partial \omega$, we obtain from (5),

$$
\forall x \in \partial \omega_{\varepsilon}, \quad \lambda_{\varepsilon} \kappa_{\varepsilon} \nu_{x}(x)=\tilde{\lambda} \kappa \nu_{y}(y) \quad \text { with } \quad y:=\frac{x-z_{0}}{\varepsilon} \in \partial \omega .
$$

For any sufficiently smooth function $f$ defined in a neighborhood of $\partial \omega_{\varepsilon}$, we set $[f]=$ $\left.f\right|_{+}-\left.f\right|_{-}$, where $\left.f\right|_{ \pm}(x)=\lim _{t \rightarrow 0} f\left(x \pm t \nu_{x}\right)$ for $x \in \partial \omega_{\varepsilon}$. Denoting by $\chi_{\partial \omega_{\varepsilon}}$ the characteristic function of $\partial \omega_{\varepsilon}$, we consider the following perturbed Stokes problem:

$$
\left\{\begin{aligned}
-\operatorname{Div}\left(2 \mu_{\varepsilon} e\left(u_{\varepsilon}\right)\right)+\nabla p_{\varepsilon} & =\lambda_{\varepsilon} \kappa_{\varepsilon} \nu_{x} \chi_{\partial \omega_{\varepsilon}} & & \text { in } \Omega, \\
\operatorname{div}\left(u_{\varepsilon}\right) & =0 & & \text { in } \Omega, \\
u_{\varepsilon} & =g & & \text { on } \partial \Omega,
\end{aligned}\right.
$$

where $\mu_{\varepsilon}$ is given by (2). Since $\lambda_{\varepsilon} \kappa_{\varepsilon} \nu_{x} \chi_{\partial \omega_{\varepsilon}} \in H^{-1}(\Omega)^{n}$ (see Lemma 3.1), there exists a unique solution $\left(u_{\varepsilon}, p_{\varepsilon}\right) \in H^{1}(\Omega)^{n} \times L_{0}^{2}(\Omega)$ to (7).

Let $(G, F) \in H^{1}(\Omega)^{n^{2}} \times L_{0}^{2}(\Omega)^{n}$ denote the Green tensors associated with the reference system (44). Precisely, for a fixed $z \in \Omega, G(\cdot, z)=\left(G_{i j}(\cdot, z)\right)_{1 \leq i, j \leq n}$ and $F(\cdot, z)=$ $\left(F_{i}(\cdot, z)\right)_{1 \leq i \leq n}$ are the solutions to

$$
\left\{\begin{aligned}
-\mu_{0} \Delta_{x} G_{i j}(x, z)+\frac{\partial F_{i}}{\partial x_{j}}(x, z) & =\delta_{i j} \delta_{z}(x) & & \text { in } \Omega, \\
\sum_{j=1}^{n} \frac{\partial G_{i j}}{\partial x_{j}}(x, z) & =0 & & \text { in } \Omega, \\
G_{i j}(x, z) & =0 & & \text { on } \partial \Omega,
\end{aligned}\right.
$$

where $\delta_{z}$ is the Dirac mass at $z$ and $\delta_{i j}$ is the Kronecker delta. Denoting by $G_{i}$ the $i$ th row of $G$, the system (8) can be rewritten as

$$
\left\{\begin{aligned}
-\operatorname{Div}_{x}\left(2 \mu_{0} e_{x}\left(G_{i}\right)(x, z)\right)+\nabla_{x} F_{i}(x, z) & =e^{i} \delta_{z}(x) & & \text { in } \Omega, \\
\operatorname{div}_{x}\left(G_{i}\right)(x, z) & =0 & & \text { in } \Omega, \\
G_{i}(x, z) & =0 & & \text { on } \partial \Omega,
\end{aligned}\right.
$$

where $e^{i}=\left(e_{j}^{i}\right)_{1 \leq j \leq n}:=\left(\delta_{i j}\right)_{1 \leq j \leq n}$. 
We also denote by $\mu$ the viscosity in the whole space containing a rescaled inclusion, i.e.

$$
\mu(y):= \begin{cases}\mu_{0} & \text { if } y \in \mathbb{R}^{n} \backslash \bar{\omega} \\ \mu_{1} & \text { if } y \in \omega\end{cases}
$$

To state our main result, we introduce the corrector $\left(\varphi^{k l}, s^{k l}\right) \in H_{\text {loc }}^{1}\left(\mathbb{R}^{n}\right)^{n} \times L_{\text {loc }}^{2}\left(\mathbb{R}^{n}\right)$, $1 \leq k, l \leq n$, the solution to

$$
\left\{\begin{aligned}
-\operatorname{Div}\left(2 \mu e\left(\varphi^{k l}\right)\right)+\nabla s^{k l} & =\left(\mu_{0}-\mu_{1}\right)\left(\left(e^{l} \nu_{k}+e^{k} \nu_{l}\right)+\frac{2}{n} \nu_{y} \delta_{k l}\right) \chi_{\partial \omega} \quad \text { in } \mathbb{R}^{n}, \\
\operatorname{div}\left(\varphi^{k l}\right) & =0 \text { in } \mathbb{R}^{n}, \\
\varphi^{k l}(y) & =O\left(|y|^{-1}\right) \quad \text { as }|y| \rightarrow \infty \\
s^{k l}(y) & =O\left(|y|^{-2}\right) \quad \text { as }|y| \rightarrow \infty
\end{aligned}\right.
$$

where $\nu_{i}$ is the $i$ th component of the exterior normal $\nu$ to $\partial \omega$. We also let $(\varphi, s) \in$ $H_{\text {loc }}^{1}\left(\mathbb{R}^{n}\right)^{n} \times L_{\text {loc }}^{2}\left(\mathbb{R}^{n}\right)$ be the solution to

$$
\left\{\begin{aligned}
-\operatorname{Div}(2 \mu e(\varphi))+\nabla s & =\kappa \nu_{y} \chi_{\partial \omega} \quad \text { in } \mathbb{R}^{n} \\
\operatorname{div}(\varphi) & =0 \text { in } \mathbb{R}^{n} \\
\varphi(y) & =O\left(|y|^{-1}\right) \quad \text { as }|y| \rightarrow \infty \\
s(y) & =O\left(|y|^{-2}\right) \quad \text { as }|y| \rightarrow \infty
\end{aligned}\right.
$$

Finally, let $\mathbf{V}$ be the symmetric, fourth-order, viscous moment tensor (in the terminology of [3]) with coefficients

$$
V_{i j k l}:=2\left(\mu_{0}-\mu_{1}\right)\left(\frac{|\omega|}{2}\left(\delta_{i k} \delta_{j l}+\delta_{i l} \delta_{j k}\right)+\int_{\omega} e\left(\varphi^{k l}\right)_{i j} d y\right), \quad 1 \leq i, j, k, l \leq n .
$$

Our main result is the following.

Theorem 2.1. For any $z \in \Omega$ at a distance $d>0$ away from $\omega_{\varepsilon}$, and for $1 \leq i \leq n$, we have

$$
\left(u_{\varepsilon}-u\right)(z)_{i}=\varepsilon^{n} e_{x}\left(G_{i}\right)\left(z, z_{0}\right): \mathbf{V} e_{x}(u)\left(z_{0}\right)+\varepsilon^{n} e_{x}\left(G_{i}\right)\left(z, z_{0}\right): K+O\left(\varepsilon^{n+\frac{1}{2}}\right) .
$$

The term $O\left(\varepsilon^{n+\frac{1}{2}}\right)$ is uniformly bounded by $C \varepsilon^{n+\frac{1}{2}}$, where the constant $C$ depends on $d$, $\mu_{0}$ and $\mu_{1}$ but is independent of $\varepsilon$. The curvature moment tensor refers to the symmetric matrix $K \in \mathbb{R}_{s}^{n \times n}$, which is given by

$$
K:=2 \tilde{\lambda}\left(\mu_{0}-\mu_{1}\right) \int_{\omega} e(\varphi) d y-\tilde{\lambda} \int_{\partial \omega} \nu_{y} \otimes \nu_{y} d \sigma_{y}
$$

where $\varphi$ is the solution to (12), and where $\nu_{y} \otimes \nu_{y}=\left(\nu_{i} \nu_{j}\right)_{1 \leq i, j \leq n} \in \mathbb{R}_{s}^{n \times n}$.

\section{REMARK 2.2 .}

(1) Existence and uniqueness for the problems (11) and (12) are established in the appendix B]

(2) The viscous moment tensor introduced in [3] is expressed somewhat differently as

$$
W_{i j k l}:=2\left(\mu_{0}-\mu_{1}\right) \int_{\omega} e_{y}\left(\phi^{k l}\right)_{i j} d y
$$


where $\phi^{k l}$ is the solution to

$$
\left\{\begin{aligned}
-\operatorname{Div}\left(2 \mu e_{y}\left(\phi^{k l}\right)\right)+\nabla p^{k l} & =0 \text { in } \mathbb{R}^{n} \\
\operatorname{div}\left(\phi^{k l}\right) & =0 \text { in } \mathbb{R}^{n} \\
\phi^{k l}(y)-y_{k} e^{l}+\frac{\delta_{k l}}{n} y & =O\left(|y|^{-1}\right) \text { as }|y| \rightarrow \infty \\
p^{k l}(y) & =O\left(|y|^{-2}\right) \quad \text { as }|y| \rightarrow \infty
\end{aligned}\right.
$$

We note that $e\left(y_{k} e^{l}-\frac{\delta_{k l}}{n} y\right)_{i j}=\frac{1}{2}\left(\delta_{i l} \delta_{j k}+\delta_{i k} \delta_{j l}\right)-\frac{1}{n} \delta_{i j} \delta_{k l}$ and that $y_{k} e^{l}-\frac{\delta_{k l}}{n} y$ is divergence free. Therefore, $\phi^{k l}(y)-y_{k} e^{l}+\frac{\delta_{k l}}{n} y$ is also a solution to (12), and uniqueness shows that it must be equal to $\varphi^{k l}$. It follows that

$$
\begin{aligned}
W_{i j k l} & =2\left(\mu_{0}-\mu_{1}\right) \int_{\omega} e_{y}\left(\phi^{k l}\right)_{i j} d y \\
& =2\left(\mu_{0}-\mu_{1}\right) \int_{\omega} e_{y}\left(\varphi^{k l}+y_{k} e^{l}-\frac{\delta_{k l}}{n} y\right)_{i j} d y \\
& =2\left(\mu_{0}-\mu_{1}\right)\left(\frac{|\omega|}{2}\left(\delta_{i l} \delta_{j k}+\delta_{i k} \delta_{j l}\right)-\frac{|\omega|}{n} \delta_{i j} \delta_{k l}+\int_{\omega} e_{y}\left(\varphi^{k l}\right)_{i j} d y\right) \\
& =V_{i j k l}-2\left(\mu_{0}-\mu_{1}\right) \frac{|\omega|}{n} \delta_{i j} \delta_{k l} .
\end{aligned}
$$

It follows that $\mathbf{V} \sigma=\mathbf{W} \sigma$, whenever $\sigma \in \mathbb{R}_{s}^{n \times n}$ with $\operatorname{tr}(\sigma)=0$. In other words, $\mathbf{W}$ and $\mathbf{V}$ coincide on the subspace of symmetric matrices with zero trace. In particular, since $u$ is divergence free, we have $\mathbf{W} e(u)\left(z_{0}\right)=\mathbf{V} e(u)\left(z_{0}\right)$.

REMARK 2.3.

(1) It will be convenient in Section 4 to express $K$ as a symmetric matrix with zero trace. To this end, we define $X \in \mathbb{R}_{s}^{n \times n}$ by

$$
X_{i j}=-\int_{\partial \omega}\left(\nu_{i} \nu_{j}-\frac{\delta_{i j}}{n}\right) d \sigma_{y} .
$$

Note that $\operatorname{tr}(X)=0$. Since $G_{i}$ is divergence free, we have

$$
e_{x}\left(G_{i}\right)\left(z_{0}, z\right): X=-e_{x}\left(G_{i}\right)\left(z_{0}, z\right):\left(\int_{\partial \omega} \nu_{y} \otimes \nu_{y} d \sigma_{y}\right)
$$

and thus, in view of (15), the matrix $K$ can be replaced by

$$
K=2 \tilde{\lambda}\left(\mu_{0}-\mu_{1}\right) \int_{\omega} e(\varphi) d y+\tilde{\lambda} X
$$

(2) Let $h_{i j}:=y_{i} e^{j}-\frac{\delta_{i j}}{n} y$. Integrating by parts on $\partial \omega$ (see (75)) we have

$$
X_{i j}=-\int_{\partial \omega}\left(\nu_{i} \nu_{j}-\frac{\delta_{i j}}{n}\right) d \sigma_{y}=-\int_{\partial \omega} e\left(h_{i j}\right) \nu_{y} \cdot \nu_{y} d \sigma_{y}=\int_{\partial \omega} \kappa \nu_{y} \cdot h_{i j} d \sigma_{y} .
$$

We note that when $\omega$ is a ball, the curvature $\kappa=\kappa_{0}$ is constant and $X=0$. Moreover, when $v \in \mathbb{C}_{0}^{\infty}\left(\mathbb{R}^{n}\right)^{n}$ and is divergence free, we see that

$$
\int_{\partial \omega} \kappa_{0} v \cdot \nu=\int_{\omega}-\operatorname{div}(v) \kappa_{0}=0
$$

so that the solution to (12) is $\varphi \equiv 0$ and $K=0$ as well. 
3. Proof of Theorem 2.1. We first note that problem (7) has a unique solution for every $\varepsilon>0$. This is a direct consequence of the general existence theorem for the Stokes problem (see [26]) and of the following result:

Lemma 3.1. Let $\varepsilon>0$ be fixed. Then $\lambda_{\varepsilon} \kappa_{\varepsilon} \nu_{x} \chi_{\partial \omega_{\varepsilon}} \in H^{-1}(\Omega)^{n}$.

Proof. Let $\psi \in H_{0}^{1}(\Omega)^{n}$ and $\bar{\psi}:=\frac{1}{\left|\omega_{\varepsilon}\right|} \int_{\omega_{\varepsilon}} \psi(x) d x$. We have

$$
\int_{\partial \omega_{\varepsilon}} \lambda_{\varepsilon} \kappa_{\varepsilon} \nu_{x} \cdot \psi d \sigma_{x}=\int_{\partial \omega_{\varepsilon}} \lambda_{\varepsilon} \kappa_{\varepsilon} \nu_{x} \cdot \bar{\psi} d \sigma_{x}+\int_{\partial \omega_{\varepsilon}} \lambda_{\varepsilon} \kappa_{\varepsilon} \nu_{x} \cdot(\psi-\bar{\psi}) d \sigma_{x}
$$

Since $\bar{\psi}$ is constant, we infer from (77) that

$$
\int_{\partial \omega_{\varepsilon}} \lambda_{\varepsilon} \kappa_{\varepsilon} \nu_{x} \cdot \bar{\psi} d \sigma_{x}=0
$$

As $\partial \omega$ is regular, $\lambda_{\varepsilon} \kappa_{\varepsilon} \nu_{x} \in L^{2}\left(\partial \omega_{\varepsilon}\right)^{n}$, and combining the Cauchy-Schwarz inequality, the trace theorem and the Poincaré-Wirtinger inequality, we obtain

$$
\begin{aligned}
\left|\int_{\partial \omega_{\varepsilon}} \lambda_{\varepsilon} \kappa_{\varepsilon} \nu_{x} \cdot(\psi-\bar{\psi}) d \sigma_{x}\right| & \leq\|\psi-\bar{\psi}\|_{L^{2}\left(\partial \omega_{\varepsilon}\right)^{n}}\left\|\lambda_{\varepsilon} \kappa_{\varepsilon} \nu_{x}\right\|_{L^{2}\left(\partial \omega_{\varepsilon}\right)^{n}} \\
& \leq c\|\psi-\bar{\psi}\|_{H^{1}\left(\omega_{\varepsilon}\right)^{n}}\left\|\lambda_{\varepsilon} \kappa_{\varepsilon} \nu_{x}\right\|_{L^{2}\left(\partial \omega_{\varepsilon}\right)^{n}} \\
& \leq c\|\nabla \psi\|_{L^{2}(\Omega)^{n}}\left\|\lambda_{\varepsilon} \kappa_{\varepsilon} \nu_{x}\right\|_{L^{2}\left(\partial \omega_{\varepsilon}\right)^{n}} \\
& \leq c\|\psi\|_{H^{1}(\Omega)^{n}}\left\|\lambda_{\varepsilon} \kappa_{\varepsilon} \nu_{x}\right\|_{L^{2}\left(\partial \omega_{\varepsilon}\right)^{n}},
\end{aligned}
$$

which proves the lemma.

The proof of Theorem 2.1 is based on the three following lemmas (we follow the approach of [14]). We start with an estimate of $u_{\varepsilon}-u$ in $H^{1}(\Omega)^{n}$.

Lemma 3.2. There exists a constant $C>0$, independent of $\varepsilon$, such that

$$
\left\|u_{\varepsilon}-u\right\|_{H^{1}(\Omega)^{n}} \leq C \varepsilon^{n / 2} .
$$

Proof. Using $u_{\varepsilon}-u$ as a test function in (4), an integration by parts yields

$$
\begin{aligned}
\int_{\Omega} 2 \mu_{\varepsilon} e(u): e\left(u_{\varepsilon}-u\right) d x= & \int_{\Omega} 2 \mu_{0} e(u): e\left(u_{\varepsilon}-u\right) d x \\
& +\int_{\Omega} 2\left(\mu_{0}-\mu_{\varepsilon}\right) e(u): e\left(u_{\varepsilon}-u\right) d x \\
= & \int_{\partial \Omega}\left(2 \mu_{0} e(u)-p I_{n}\right) \nu_{x} \cdot\left(u_{\varepsilon}-u\right) d \sigma_{x} \\
& +2 \int_{\omega_{\varepsilon}}\left(\mu_{0}-\mu_{1}\right) e(u): e\left(u_{\varepsilon}-u\right) d x \\
= & 2 \int_{\omega_{\varepsilon}}\left(\mu_{0}-\mu_{1}\right) e(u): e\left(u_{\varepsilon}-u\right) d x
\end{aligned}
$$


Next, choosing $u_{\varepsilon}-u$ as a test function in (7) yields

$$
\begin{aligned}
\int_{\Omega} 2 \mu_{\varepsilon} e\left(u_{\varepsilon}\right): e\left(u_{\varepsilon}-u\right) d x= & \int_{\partial \Omega}\left(2 \mu_{0} e\left(u_{\varepsilon}\right)-p_{\varepsilon} I_{n}\right) \nu_{x} \cdot\left(u_{\varepsilon}-u\right) d \sigma_{x} \\
& +\int_{\partial \omega_{\varepsilon}} \lambda_{\varepsilon} \kappa_{\varepsilon} \nu_{x} \cdot\left(u_{\varepsilon}-u\right) d \sigma_{x} \\
= & \int_{\partial \omega_{\varepsilon}} \lambda_{\varepsilon} \kappa_{\varepsilon} \nu_{x} \cdot\left(u_{\varepsilon}-u\right) d \sigma_{x} .
\end{aligned}
$$

Combining these equalities, we obtain

$$
\begin{aligned}
\int_{\Omega} 2 \mu_{\varepsilon}\left|e\left(u_{\varepsilon}-u\right)\right|^{2} d x= & \int_{\partial \omega_{\varepsilon}} \lambda_{\varepsilon} \kappa_{\varepsilon} \nu_{x} \cdot\left(u_{\varepsilon}-u\right) d \sigma_{x} \\
& +\int_{\omega_{\varepsilon}} 2\left(\mu_{1}-\mu_{0}\right) e(u): e\left(u_{\varepsilon}-u\right) d x .
\end{aligned}
$$

Let $w_{\varepsilon}:=u_{\varepsilon}-u$ and $\bar{w}_{\varepsilon}:=\frac{1}{\left|\omega_{\varepsilon}\right|} \int_{\omega_{\varepsilon}} w_{\varepsilon}(x) d x$. It follows from (77) that

$$
\int_{\partial \omega_{\varepsilon}} \lambda_{\varepsilon} \kappa_{\varepsilon} \nu_{x} \cdot \bar{w}_{\varepsilon} d \sigma_{x}=0
$$

Recalling (6) , the fact that $\tilde{\lambda} \kappa \nu_{y} \in L^{2}(\partial \omega)^{n}$, and changing variables to $y=\left(x-z_{0}\right) / \varepsilon$, we apply the trace theorem and the Poincaré-Wirtinger inequality to obtain

$$
\begin{aligned}
\left|\int_{\partial \omega_{\varepsilon}} \lambda_{\varepsilon} \kappa_{\varepsilon} \nu_{x} \cdot\left(w_{\varepsilon}-\bar{w}_{\varepsilon}\right) d \sigma_{x}\right| & =\varepsilon^{n-1}\left|\int_{\partial \omega} \tilde{\lambda} \kappa \nu_{y} \cdot\left(w_{\varepsilon}\left(\varepsilon y+z_{0}\right)-\bar{w}_{\varepsilon}\right) d \sigma_{y}\right| \\
& \left.\leq c \varepsilon^{n-1} \| w_{\varepsilon}\left(\varepsilon y+z_{0}\right)-\bar{w}_{\varepsilon}\right) \|_{L^{2}(\partial \omega)^{n}} \\
& \left.\leq c \varepsilon^{n-1} \| w_{\varepsilon}\left(\varepsilon y+z_{0}\right)-\bar{w}_{\varepsilon}\right) \|_{H^{1}(\omega)^{n}} \\
& \leq c \varepsilon^{n-1}\left\|\nabla_{y}\left(w_{\varepsilon}\left(\varepsilon y+z_{0}\right)\right)\right\|_{L^{2}(\omega)^{n^{2}}} .
\end{aligned}
$$

Further, invoking the Korn inequality, we have

$$
\begin{aligned}
\left\|\nabla_{y}\left(w_{\varepsilon}\left(\varepsilon y+z_{0}\right)\right)\right\|_{L^{2}(\omega) n^{2}} & =\varepsilon^{\frac{2-n}{2}}\left\|\nabla_{x} w_{\varepsilon}\right\|_{L^{2}\left(\omega_{\varepsilon}\right)^{n^{2}}} \leq \varepsilon^{\frac{2-n}{2}}\left\|\nabla_{x} w_{\varepsilon}\right\|_{L^{2}(\Omega)^{n^{2}}} \\
& \leq C \varepsilon^{\frac{2-n}{2}}\left\|e_{x} w_{\varepsilon}\right\|_{L^{2}(\Omega)^{n^{2}}},
\end{aligned}
$$

where the constant $C$ is independent of $\varepsilon$. In view of (23), we obtain

$$
\left|\int_{\partial \omega_{\varepsilon}} \lambda_{\varepsilon} \kappa_{\varepsilon} \nu_{x} \cdot\left(u_{\varepsilon}-u\right) d \sigma_{x}\right| \leq C \varepsilon^{\frac{n}{2}}\left\|e_{x}\left(u_{\varepsilon}-u\right)\right\|_{L^{2}(\Omega)^{n^{2}}} .
$$

Combining (22), the Cauchy-Schwarz inequality and the fact that $u$ is bounded in $\Omega$ shows that

$$
\begin{aligned}
\int_{\Omega} \mu_{\varepsilon}\left|e\left(u_{\varepsilon}-u\right)\right|^{2} d x \leq & \left|\mu_{1}-\mu_{0}\right|\|e(u)\|_{L^{2}\left(\omega_{\varepsilon}\right)^{n^{2}}}\left\|e\left(u_{\varepsilon}-u\right)\right\|_{L^{2}\left(\omega_{\varepsilon}\right)^{n^{2}}} \\
& +C \varepsilon^{\frac{n}{2}}\left\|e_{x}\left(u_{\varepsilon}-u\right)\right\|_{L^{2}(\Omega)^{n^{2}}} \\
\leq & C \varepsilon^{\frac{n}{2}}\left\|e\left(u_{\varepsilon}-u\right)\right\|_{L^{2}(\Omega)^{n^{2}}}
\end{aligned}
$$

which yields the desired result. 
We next consider

$$
\Omega_{\varepsilon}:=\left\{\frac{x-z_{0}}{\varepsilon} \mid x \in \Omega\right\}
$$

Let $\left(V_{\varepsilon}, Q_{\varepsilon}\right) \in\left(H^{1}\left(\Omega_{\varepsilon}\right)^{n} \times L_{0}^{2}\left(\Omega_{\varepsilon}\right)\right)$ be the unique solution to

$$
\left\{\begin{aligned}
-\operatorname{Div}_{y}\left(2 \mu e_{y}\left(V_{\varepsilon}\right)\right)+\nabla_{y} Q_{\varepsilon} & =\left(\tilde{\lambda} \kappa \nu_{y}-2\left(\mu_{1}-\mu_{0}\right) e(u)\left(z_{0}\right) \nu_{y}\right) \chi_{\partial \omega} & & \text { in } \Omega_{\varepsilon} \\
\operatorname{div}\left(V_{\varepsilon}\right) & =0 & & \text { in } \Omega_{\varepsilon} \\
V_{\varepsilon} & =0 & & \text { on } \partial \Omega_{\varepsilon}
\end{aligned}\right.
$$

where $\mu=\mu(y)$ is given by (10).

Lemma 3.3. There exists a constant $C>0$, independent of $\varepsilon$, such that

$$
\left\|e_{y}\left(u_{\varepsilon}\left(\varepsilon y+z_{0}\right)-u\left(\varepsilon y+z_{0}\right)-\varepsilon V_{\varepsilon}(y)\right)\right\|_{L^{2}\left(\Omega_{\varepsilon}\right)^{n^{2}}} \leq C \varepsilon^{2} .
$$

Proof. Let $Z_{\varepsilon}(y):=u_{\varepsilon}\left(\varepsilon y+z_{0}\right)-u\left(\varepsilon y+z_{0}\right)-\varepsilon V_{\varepsilon}(y)$, and form

$$
\begin{aligned}
\int_{\Omega_{\varepsilon}} 2 \mu\left|e_{y}\left(Z_{\varepsilon}\right)\right|^{2} d y= & \int_{\Omega_{\varepsilon}} 2 \mu e_{y}\left(u_{\varepsilon}\left(\varepsilon y+z_{0}\right)\right): e_{y}\left(Z_{\varepsilon}\right) d y \\
& -\int_{\Omega_{\varepsilon}} 2 \mu e_{y}\left(u\left(\varepsilon y+z_{0}\right)\right): e_{y}\left(Z_{\varepsilon}\right) d y \\
& -\varepsilon \int_{\Omega_{\varepsilon}} 2 \mu e_{y}\left(V_{\varepsilon}\right): e_{y}\left(Z_{\varepsilon}\right) d y
\end{aligned}
$$

For $x \in \Omega$, we set $z_{\varepsilon}(x):=Z_{\varepsilon}\left(\frac{x-z_{0}}{\varepsilon}\right)=Z_{\varepsilon}(y)$, and we examine each term in the righthand side of the previous equality.

From equations (6) and since $Z_{\varepsilon}$ is divergence free and $z_{\varepsilon}=0$ on $\partial \Omega$, it follows that the first term can be rewritten as

$$
\begin{aligned}
\int_{\Omega_{\varepsilon}} 2 \mu e_{y}\left(u_{\varepsilon}\left(\varepsilon y+z_{0}\right)\right): e_{y}\left(Z_{\varepsilon}\right) d y= & \varepsilon^{2-n} \int_{\Omega} 2 \mu_{\varepsilon} e_{x}\left(u_{\varepsilon}\right): e_{x}\left(z_{\varepsilon}\right) d x \\
= & \left.\varepsilon^{2-n} \int_{\partial \Omega} 2 \mu_{\varepsilon} e_{x}\left(u_{\varepsilon}\right)-p_{\varepsilon} I_{n}\right) \nu_{x} \cdot z_{\varepsilon} d \sigma_{x} \\
& +\varepsilon^{2-n} \int_{\partial \omega_{\varepsilon}} \lambda_{\varepsilon} \kappa_{\varepsilon} \nu_{x} \cdot z_{\varepsilon} d \sigma_{x} \\
= & \varepsilon \int_{\partial \omega} \tilde{\lambda} \kappa \nu_{y} \cdot Z_{\varepsilon} d \sigma_{y} .
\end{aligned}
$$


For the second term, we have

$$
\begin{aligned}
\int_{\Omega_{\varepsilon}} 2 \mu e_{y}\left(u\left(\varepsilon y+z_{0}\right)\right): e_{y}\left(Z_{\varepsilon}\right) d y= & \varepsilon^{2-n} \int_{\Omega} 2 \mu_{\varepsilon} e_{x}(u): e_{x}\left(z_{\varepsilon}\right) d x \\
= & \varepsilon^{2-n} \int_{\Omega} 2 \mu_{0} e_{x}(u): e_{x}\left(z_{\varepsilon}\right) d x \\
& +\varepsilon^{2-n} \int_{\omega_{\varepsilon}} 2\left(\mu_{1}-\mu_{0}\right) e_{x}(u): e_{x}\left(z_{\varepsilon}\right) d x \\
= & \varepsilon \int_{\omega} 2\left(\mu_{1}-\mu_{0}\right) e_{x}(u)\left(\varepsilon y+z_{0}\right): e_{y}\left(Z_{\varepsilon}\right) d y .
\end{aligned}
$$

Finally, using $Z_{\varepsilon}$ as a test function in (24), we see that

$$
\int_{\Omega_{\varepsilon}} 2 \mu e_{y}\left(V_{\varepsilon}\right): e_{y}\left(Z_{\varepsilon}\right) d y=\int_{\partial \omega} \tilde{\lambda} \kappa \nu_{y} \cdot Z_{\varepsilon} d \sigma_{y}-2\left(\mu_{1}-\mu_{0}\right) \int_{\partial \omega} e_{x}(u)\left(z_{0}\right) \nu_{y} \cdot Z_{\varepsilon} d \sigma_{y} .
$$

It follows that

$$
\int_{\Omega_{\varepsilon}} 2 \mu\left|e_{y}\left(Z_{\varepsilon}\right)\right|^{2} d y=\varepsilon \int_{\omega} 2\left(\mu_{0}-\mu_{1}\right)\left[e_{x}(u)\left(\varepsilon y+z_{0}\right)-e_{x}(u)\left(z_{0}\right)\right]: e_{y}\left(Z_{\varepsilon}\right) d y .
$$

Since $u$ is smooth inside $\Omega$, $\left\|e_{x}(u)\left(\varepsilon y+z_{0}\right)-e_{x}(u)\left(z_{0}\right)\right\|_{L^{\infty}(\omega)} \leq C \varepsilon$, and we conclude that

$$
\left\|e_{y}\left(Z_{\varepsilon}\right)\right\|_{L^{2}\left(\Omega_{\varepsilon}\right)^{n^{2}}} \leq C \varepsilon^{2} .
$$

Let $(V, Q) \in H^{1}\left(\mathbb{R}^{n}\right)_{\text {loc }}^{n} \times L^{2}\left(\mathbb{R}^{n}\right)_{\text {loc }}$ be the unique solution to

$$
\left\{\begin{aligned}
-\operatorname{Div}_{y}\left(2 \mu e_{y}(V)\right)+\nabla_{y} q & =\left(\tilde{\lambda} \kappa \nu_{y}-2\left(\mu_{1}-\mu_{0}\right) e(u)\left(z_{0}\right) \nu_{y}\right) \chi_{\partial \omega} & & \text { in } \mathbb{R}^{n}, \\
\operatorname{div}(V) & =0 & & \text { in } \mathbb{R}^{n}, \\
V(y) & =O\left(|y|^{-1}\right) & & \text { as }|y| \rightarrow \infty, \\
Q(y) & =O\left(|y|^{-2}\right) & & \text { as }|y| \rightarrow \infty .
\end{aligned}\right.
$$

Lemma 3.4. Assume that $n=2$ or $n=3$. There exists a constant $C$, independent of $\varepsilon$, such that

$$
\left\|e_{y}\left(u_{\varepsilon}\left(\varepsilon y+z_{0}\right)-u\left(\varepsilon y+z_{0}\right)-\varepsilon V(y)\right)\right\|_{L^{2}\left(\Omega_{\varepsilon}\right)^{n^{2}}} \leq C \varepsilon^{3 / 2} .
$$

Proof. According to Lemma 3.3, it suffices to show that

$$
\left\|e_{y}\left(V_{\varepsilon}-V\right)(y)\right\|_{L^{2}\left(\Omega_{\varepsilon}\right)^{n^{2}}} \leq C \varepsilon^{1 / 2} .
$$

We set

$$
W_{\varepsilon}:=V_{\varepsilon}-V, \quad R_{\varepsilon}:=Q_{\varepsilon}-Q .
$$

Then, $W_{\varepsilon} \in H^{1}\left(\Omega_{\varepsilon}\right)^{n}, R_{\varepsilon} \in L_{0}^{2}\left(\Omega_{\varepsilon}\right)$ solve

$$
\left\{\begin{aligned}
-\operatorname{Div}_{y}\left(2 \mu e_{y}\left(W_{\varepsilon}\right)\right)+\nabla_{y} R_{\varepsilon} & =0 & & \text { in } \Omega_{\varepsilon}, \\
\operatorname{div}\left(W_{\varepsilon}\right) & =0 & & \text { in } \Omega_{\varepsilon}, \\
W_{\varepsilon} & =-V & & \text { on } \partial \Omega_{\varepsilon} .
\end{aligned}\right.
$$


Changing variables to $x=\varepsilon y+z_{0}$, we set for $x \in \Omega$,

$$
w_{\varepsilon}(x):=W_{\varepsilon}\left(\frac{x-z_{0}}{\varepsilon}\right), \quad r_{\varepsilon}(x):=R_{\varepsilon}\left(\frac{x-z_{0}}{\varepsilon}\right), \quad v(x):=V\left(\frac{x-z_{0}}{\varepsilon}\right) .
$$

We compute

$$
\begin{aligned}
\int_{\Omega_{\varepsilon}} 2 \mu e_{y}\left(W_{\varepsilon}\right): e_{y}\left(W_{\varepsilon}\right) d y & =-\int_{\partial \Omega_{\varepsilon}}\left(2 \mu e_{y}\left(W_{\varepsilon}\right)-R_{\varepsilon} I_{n}\right) \nu_{y} \cdot V d \sigma_{y} \\
& =-\varepsilon^{1-n} \int_{\partial \Omega}\left(2 \varepsilon \mu_{\varepsilon} e_{x}\left(w_{\varepsilon}\right)-r_{\varepsilon} I_{n}\right) \nu_{x} \cdot v d \sigma_{x}
\end{aligned}
$$

Since $v$ is divergence free in $\Omega$, there exists $\psi \in H^{1}(\Omega)^{n}$ such that (see e.g. [26]),

$$
\operatorname{div}(\psi)=0 \text { in } \Omega, \quad \psi=v \text { on } \partial \Omega
$$

In addition, $\psi$ satisfies

$$
\|\psi\|_{H^{1}(\Omega)^{n}} \leq C\|v\|_{H^{\frac{1}{2}}(\partial \Omega)^{n}},
$$

for some constant $C>0$ independent of $v$. The Cauchy-Schwarz inequality, implies

$$
\begin{aligned}
\left|\int_{\Omega_{\varepsilon}} 2 \mu e_{y}\left(W_{\varepsilon}\right): e_{y}\left(W_{\varepsilon}\right) d y\right| & =\varepsilon^{1-n}\left|\int_{\partial \Omega}\left(2 \varepsilon \mu_{\varepsilon} e_{x}\left(w_{\varepsilon}\right)-r_{\varepsilon} I_{n}\right) \nu_{x} \cdot \psi d \sigma_{x}\right| \\
& =\varepsilon^{1-n}\left|\int_{\Omega} 2 \varepsilon \mu_{\varepsilon} e_{x}\left(w_{\varepsilon}\right): e_{x}(\psi) d x\right| \\
& \leq \varepsilon^{2-n}\left\|e_{x}\left(w_{\varepsilon}\right)\right\|_{L^{2}(\Omega)^{n^{2}}}\left\|e_{x}(\psi)\right\|_{L^{2}(\Omega)^{n^{2}}} \\
& \leq C \varepsilon^{2-n}\left\|e_{x}\left(w_{\varepsilon}\right)\right\|_{L^{2}(\Omega)^{n^{2}}}\|v\|_{H^{\frac{1}{2}}(\partial \Omega)^{n}} \\
& \leq C \varepsilon^{1-\frac{n}{2}}\left\|e_{y}\left(W_{\varepsilon}\right)\right\|_{L^{2}\left(\Omega_{\varepsilon}\right)^{n^{2}}}\|v\|_{H^{\frac{1}{2}}(\partial \Omega)^{n}} .
\end{aligned}
$$

Moreover, invoking Lemma B.2 and the corresponding decay (779) of $V$, we see that

$$
\|v\|_{H^{\frac{1}{2}}(\partial \Omega)^{n}} \leq C \varepsilon^{\frac{1}{2}}\|V\|_{H^{\frac{1}{2}}\left(\partial \Omega_{\varepsilon}\right)^{n}} \leq C \varepsilon,
$$

from which we deduce the estimate (27)

Proof of Theorem 2.1. Let $z \in \Omega$ at a distance $d>0$ away from $\omega_{\varepsilon}$.

From the equations (9) and (44), we have for $1 \leq i \leq n$,

$$
\begin{aligned}
u_{i}(z) & =-\int_{\Omega} \operatorname{Div}\left(2 \mu_{0} e_{x}\left(G_{i}\right)\right) \cdot u d x+\int_{\Omega} \nabla_{x} F_{i} \cdot u d x \\
& =\int_{\Omega} 2 \mu_{0} e_{x}\left(G_{i}\right): e_{x}(u) d x-\int_{\partial \Omega} 2 \mu_{0} e_{x}\left(G_{i}\right) \nu_{x} \cdot u d \sigma_{x}+\int_{\partial \Omega} F_{i} \nu_{x} \cdot u d \sigma_{x} \\
& =\int_{\Omega} 2 \mu_{0} e_{x}\left(G_{i}\right): e_{x}(u) d x-\int_{\partial \Omega}\left(2 \mu_{0} e_{x}\left(G_{i}\right)-F_{i} I_{n}\right) \nu_{x} \cdot g d \sigma_{x} \\
& =-\int_{\partial \Omega}\left(2 \mu_{0} e_{x}\left(G_{i}\right)-F_{i} I_{n}\right) \nu_{x} \cdot g d \sigma_{x} .
\end{aligned}
$$


Similarly, equations (9), (4) and (30) yield for $1 \leq i \leq n$,

$$
\begin{aligned}
\left(u_{\varepsilon}\right)_{i}(z) & =-\int_{\Omega} \operatorname{Div}\left(2 \mu_{0} e_{x}\left(G_{i}\right)\right) \cdot u_{\varepsilon} d x+\int_{\Omega} \nabla_{x} F_{i} \cdot u_{\varepsilon} d x \\
& =\int_{\Omega} 2 \mu_{0} e_{x}\left(G_{i}\right): e_{x}\left(u_{\varepsilon}\right) d x-\int_{\partial \Omega}\left(2 \mu_{0} e_{x}\left(G_{i}\right)-F_{i} I_{n}\right) \nu_{x} \cdot g d \sigma_{x} \\
& =u_{i}(z)+\int_{\Omega} 2 \mu_{0} e_{x}\left(G_{i}\right): e_{x}\left(u_{\varepsilon}\right) d x \\
& =u_{i}(z)+\int_{\omega_{\varepsilon}} 2\left(\mu_{0}-\mu_{1}\right) e_{x}\left(G_{i}\right): e_{x}\left(u_{\varepsilon}\right) d x+\int_{\Omega} 2 \mu_{\varepsilon} e_{x}\left(G_{i}\right): e_{x}\left(u_{\varepsilon}\right) d x .
\end{aligned}
$$

As in (21), the last term of the right-hand side of the previous equality can be rewritten as

$$
\int_{\Omega} 2 \mu_{\varepsilon} e_{x}\left(G_{i}\right): e_{x}\left(u_{\varepsilon}\right) d x=\int_{\partial \omega_{\varepsilon}} \lambda_{\varepsilon} \kappa_{\varepsilon} \nu_{x} \cdot G_{i} d \sigma_{x}
$$

and thus,

$$
\left(u_{\varepsilon}-u\right)(z)_{i}=\int_{\omega_{\varepsilon}} 2\left(\mu_{0}-\mu_{1}\right) e_{x}\left(G_{i}\right)(x, z): e_{x}\left(u_{\varepsilon}\right) d x+\int_{\partial \omega_{\varepsilon}} \lambda_{\varepsilon} \kappa_{\varepsilon} \nu_{x} \cdot G_{i}(x, z) d \sigma_{x} .
$$

Since $z$ is away from $z_{0}$, the function $x \mapsto G_{i}(x, z)$ is smooth in a neighborhood of $z_{0}$, so that the Taylor expansion of $G_{i}\left(\varepsilon y+z_{0}, z\right)$ about $z_{0}$ and relations (6) and (75) yield

$$
\begin{aligned}
\int_{\partial \omega_{\varepsilon}} \lambda_{\varepsilon} \kappa_{\varepsilon} \nu_{x} \cdot G_{i}(x, z) d \sigma_{x} & =\varepsilon^{n-1} \int_{\partial \omega} \tilde{\lambda} \kappa \nu_{y} \cdot G_{i}\left(\varepsilon y+z_{0}, z\right) d \sigma_{y} \\
= & \varepsilon^{n-1} \int_{\partial \omega} \tilde{\lambda} \kappa \nu_{y} \cdot G_{i}\left(z_{0}, z\right) d \sigma_{y} \\
& +\varepsilon^{n} \int_{\partial \omega} \tilde{\lambda} \kappa \nu_{y} \cdot \nabla_{x} G_{i}\left(z_{0}, z\right) y d \sigma_{y}+O\left(\varepsilon^{n+1}\right) \\
= & \varepsilon^{n} \int_{\partial \omega} \tilde{\lambda} \kappa \nu_{y} \cdot \nabla_{x} G_{i}\left(z_{0}, z\right) y d \sigma_{y}+O\left(\varepsilon^{n+1}\right) \\
& =\varepsilon^{n} \tilde{\lambda} \int_{\partial \omega} \operatorname{div}_{\partial \omega}\left(\nabla_{x} G_{i}\left(z_{0}, z\right) y\right) d \sigma_{y}+O\left(\varepsilon^{n+1}\right),
\end{aligned}
$$

where the term $O\left(\varepsilon^{n+1}\right)$ is uniformly bounded by $C \varepsilon^{n+1}$ for some $C$ independent of $\varepsilon$. Since $G_{i}$ is divergence free, we deduce from (76) that

$$
\begin{aligned}
\int_{\partial \omega_{\varepsilon}} \lambda_{\varepsilon} \kappa_{\varepsilon} \nu_{x} \cdot G_{i}(x, z) d \sigma_{x} & =-\varepsilon^{n} \tilde{\lambda} \int_{\partial \omega} \nabla_{x} G_{i}\left(z_{0}, z\right) \nu_{y} \cdot \nu_{y} d \sigma_{y}+O\left(\varepsilon^{n+1}\right) \\
& =-\varepsilon^{n} \tilde{\lambda} e_{x}\left(G_{i}\right)\left(z_{0}, z\right): \int_{\partial \omega} \nu_{y} \otimes \nu_{y} d \sigma_{y}+O\left(\varepsilon^{n+1}\right) .
\end{aligned}
$$

We set

$$
R_{\varepsilon}(y):=u_{\varepsilon}\left(\varepsilon y+z_{0}\right)-u\left(\varepsilon y+z_{0}\right)-\varepsilon V(y) \quad \text { and } \quad r_{\varepsilon}(x):=R_{\varepsilon}\left(\frac{x-z_{0}}{\varepsilon}\right)=R_{\varepsilon}(y) .
$$


The first term of the right-hand side of (31) becomes

$$
\begin{aligned}
\int_{\omega_{\varepsilon}} 2\left(\mu_{0}-\mu_{1}\right) e_{x}\left(G_{i}\right)(x, z): & e_{x}\left(u_{\varepsilon}\right) d x=\int_{\omega_{\varepsilon}} 2\left(\mu_{0}-\mu_{1}\right) e_{x}\left(G_{i}\right)(x, z): e_{x}\left(r_{\varepsilon}\right) d x \\
& +\int_{\omega_{\varepsilon}} 2\left(\mu_{0}-\mu_{1}\right) e_{x}\left(G_{i}\right)(x, z): e_{x}\left(u+\varepsilon V\left(\frac{x-z_{0}}{\varepsilon}\right)\right) d x .
\end{aligned}
$$

Since $z$ is far from $z_{0}, e_{x}\left(G_{i}\right)\left(\varepsilon y+z_{0}, z\right)$ is uniformly bounded for $\varepsilon$ small enough. In view of Lemma 3.4, it follows that

$$
\begin{aligned}
& \left|\int_{\omega_{\varepsilon}} 2\left(\mu_{0}-\mu_{1}\right) e_{x}\left(G_{i}\right)(x, z): e_{x}\left(r_{\varepsilon}\right) d x\right| \\
& \quad=\varepsilon^{n-1}\left|\int_{\omega} 2\left(\mu_{0}-\mu_{1}\right) e_{x}\left(G_{i}\right)\left(\varepsilon y+z_{0}, z\right): e_{y}\left(R_{\varepsilon}\right) d y\right| \\
& \quad \leq c \varepsilon^{n+\frac{1}{2}} .
\end{aligned}
$$

Thus, considering (31) and (32), we obtain

$$
\begin{aligned}
\left(u_{\varepsilon}-u\right)(z)_{i}= & 2\left(\mu_{0}-\mu_{1}\right) \int_{\omega_{\varepsilon}} e_{x}\left(G_{i}\right)(x, z): e_{x}\left(u+\varepsilon V\left(\frac{x-z_{0}}{\varepsilon}\right)\right) d x \\
& -\varepsilon^{n} \tilde{\lambda} e_{x}\left(G_{i}\right)\left(z_{0}, z\right): \int_{\partial \omega} \nu_{y} \otimes \nu_{y} d \sigma_{y}+O\left(\varepsilon^{n+\frac{1}{2}}\right),
\end{aligned}
$$

where the term $O\left(\varepsilon^{n+\frac{1}{2}}\right)$ is uniformly bounded by $C \varepsilon^{n+\frac{1}{2}}$, for some constant $C$ that depends on $d, \mu_{0}$ and $\mu_{1}$, but is independent of $\varepsilon$. Further, a Taylor expansion of $e_{x}\left(G_{i}\right)(\varepsilon y+$ $\left.z_{0}, z\right)$ yields

$$
\begin{aligned}
\int_{\omega_{\varepsilon}} & e_{x}\left(G_{i}\right)(x, z): e_{x}\left(u+\varepsilon V\left(\frac{x-z_{0}}{\varepsilon}\right)\right) d x \\
& =\varepsilon^{n} \int_{\omega} e_{x}\left(G_{i}\right)\left(\varepsilon y+z_{0}, z\right):\left(e_{x}(u)\left(\varepsilon y+z_{0}\right)+e_{y}(V)(y)\right) d y . \\
& =\varepsilon^{n} \int_{\omega} e_{x}\left(G_{i}\right)\left(z_{0}, z\right):\left(e_{x}(u)\left(z_{0}\right)+e_{y}(V)(y)\right) d y+O\left(\varepsilon^{n+1}\right) .
\end{aligned}
$$

Noting that the pair $(V, Q)$ solution of (26) is an affine function of $e_{x}(u)\left(z_{0}\right)$, and can be rewritten as

$$
V(y)=\sum_{k, l=1}^{n} e_{x}(u)\left(z_{0}\right)_{k l} \varphi^{k l}(y)+\tilde{\lambda} \varphi(y) \text { and } Q(y)=\sum_{k, l=1}^{n} e_{x}(u)\left(z_{0}\right)_{k l} s^{k l}(y)+\tilde{\lambda} s(y),
$$

where $\left(\varphi^{k l}, s^{k l}\right)$ and $(\varphi, s)$ are solutions to (11) and (12) respectively, the last equality yields

$$
\begin{aligned}
& \int_{\omega_{\varepsilon}} e_{x}\left(G_{i}\right)(x, z): e_{x}\left(u+\varepsilon v\left(\frac{x-z_{0}}{\varepsilon}\right)\right) d x \\
& =\varepsilon^{n} \int_{\omega} e_{x}\left(G_{i}\right)\left(z_{0}, z\right):\left(e_{x}(u)\left(z_{0}\right)+\sum_{k, l=1}^{n} e_{x}(u)\left(z_{0}\right)_{k l} e\left(\varphi^{k l}\right)\right) d y \\
& \quad+\tilde{\lambda} \varepsilon^{n} \int_{\omega} e_{x}\left(G_{i}\right)\left(z_{0}, z\right): e(\varphi) d y+O\left(\varepsilon^{n+1}\right) .
\end{aligned}
$$


Finally, using (33) and (35), we obtain the desired asymptotic

$$
\begin{array}{r}
\left(u_{\varepsilon}-u\right)(z)_{i}=2\left(\mu_{0}-\mu_{1}\right) \varepsilon^{n} e_{x}\left(G_{i}\right)\left(z_{0}, z\right): \int_{\omega}\left(e_{x}(u)\left(z_{0}\right)+\sum_{k, l=1}^{n} e_{x}(u)\left(z_{0}\right)_{k l} e\left(\varphi^{k l}\right)\right) d y \\
+\varepsilon^{n} e_{x}\left(G_{i}\right)\left(z_{0}, z\right):\left(2 \tilde{\lambda}\left(\mu_{0}-\mu_{1}\right) \int_{\omega} e(\varphi) d y-\tilde{\lambda} \int_{\partial \omega} \nu_{y} \otimes \nu_{y} d \sigma_{y}\right)+O\left(\varepsilon^{n+\frac{1}{2}}\right),
\end{array}
$$

which, using the notation (13) and (15), reduces to (14).

4. Curvature moment tensor for ellipses and ellipsoids. In [3], based on layer potential techniques, the authors have derived an analytic expression of the viscous moment tensor $\mathbf{V}$ in the case where $\omega$ is an ellipse or ellipsoid.

In this section we use the same techniques to determine the curvature moment tensor $K$ for ellipses and ellipsoids. We first state a general result that holds for inclusions that satisfy a particular geometric assumption. Ellipses and ellipsoids fall in this class 7 .

The components of the fundamental Stokes tensor $\boldsymbol{\Gamma}$ and those of the associated pressure vector $P$, which determine the fundamental solution $(\boldsymbol{\Gamma}, P)$ of the Stokes system in $\mathbb{R}^{n}$, are given by (see for instance [19])

$$
\left\{\begin{aligned}
\Gamma_{i j}(x, y) & =\frac{1}{8 \pi}\left(\frac{\delta_{i j}}{|x-y|}+\frac{\left(x_{i}-y_{i}\right)\left(x_{j}-y_{j}\right)}{|x-y|^{3}}\right), \\
P_{i}(x, y) & =\frac{1}{4 \pi} \frac{x_{i}-y_{i}}{|y-z|^{3}}
\end{aligned}\right.
$$

for $n=3$, and

$$
\left\{\begin{aligned}
\Gamma_{i j}(x, y) & =-\frac{1}{4 \pi}\left(\delta_{i j} \log (|x-y|)-\frac{\left(x_{i}-y_{i}\right)\left(x_{j}-y_{j}\right)}{|x-y|^{2}}\right), \\
P_{i}(x, y) & =\frac{1}{2 \pi} \frac{x_{i}-y_{i}}{|y-z|^{2}}
\end{aligned}\right.
$$

for $n=2$. The single layer potentials of a function $\psi \in L^{2}(\partial \omega)^{n}$ on $\partial \omega$ associated with the viscosity $\mu_{0}$ are defined for $x \in \mathbb{R}^{n} \backslash \partial \omega$ by

$$
\left\{\begin{aligned}
\mathcal{S}_{0} \psi(x) & :=\frac{1}{\mu_{0}} \int_{\partial \omega} \boldsymbol{\Gamma}(x, y) \psi(y) d \sigma_{y} \\
\mathcal{Q}_{0} \psi(x) & :=\int_{\partial \omega} P(x, y) \cdot \psi(y) d \sigma_{y} .
\end{aligned}\right.
$$

Analogously, we denote by $\mathcal{S}_{1}$ and $\mathcal{Q}_{1}$ the single layer potentials on $\partial \omega$ associated with the viscosity $\mu_{1}$ (note that $\mathcal{Q}_{1}=\mathcal{Q}_{0}$ ). Then, for all $\psi \in L^{2}(\partial \omega)^{n}$, the pair $\left(\mathcal{S}_{i} \psi, \mathcal{Q}_{i} \psi\right)$ is a solution to the Stokes system with viscosity $\mu_{i}$ in $\mathbb{R}^{n} \backslash \partial \omega$.

We also denote by $\partial / \partial \nu_{i}$ the conormal derivative associated with the viscosity $\mu_{i}$, i.e.

$$
\frac{\partial u}{\partial \nu_{i}}:=\left(2 \mu_{i} e(u)-p I_{n}\right) \nu_{y}
$$


where $p$ is the pressure associated with the velocity $u$. Then, we have the following well-known jump relation (see e.g. [3]) for every $\psi \in L^{2}(\partial \omega)^{n}$ :

$$
\left.\frac{\partial\left(\mathcal{S}_{i} \psi\right)}{\partial \nu_{i}}\right|_{-}-\left.\frac{\partial\left(\mathcal{S}_{i} \psi\right)}{\partial \nu_{i}}\right|_{+}=\psi \text { a.e. on } \partial \omega, i=0,1 \text {. }
$$

Moreover, $\mathcal{S}_{i} \psi$ is continuous across $\partial \omega$. Consider the system of integral equations:

$$
\left\{\begin{array}{c}
\left.\mathcal{S}_{0} g\right|_{+}-\left.\mathcal{S}_{1} f\right|_{-}=\left.0\right|_{\partial \omega} \\
\left.\frac{\partial\left(\mathcal{S}_{0} g\right)}{\partial \nu_{0}}\right|_{+}-\left.\frac{\partial\left(\mathcal{S}_{1} f\right)}{\partial \nu_{1}}\right|_{-}=\left.\kappa \nu\right|_{\partial \omega} .
\end{array}\right.
$$

Then, the solution $\varphi$ of (12) is given by

$$
\varphi= \begin{cases}\mathcal{S}_{0} g & \text { in } \mathbb{R}^{n} \backslash \bar{\omega}, \\ \mathcal{S}_{1} f & \text { in } \omega,\end{cases}
$$

where $(f, g) \in L^{2}(\partial \omega)^{n} \times L^{2}(\partial \omega)^{n}$ is the unique solution to (411) (see [3]).

REMARK 4.1. If $h$ is a divergence-free linear vector-valued function, we have the following integration by parts formula:

$$
\int_{\partial \omega} \varphi \cdot\left(\frac{\partial h}{\partial \nu_{0}}-\frac{\partial h}{\partial \nu_{1}}\right) d \sigma_{y}=\int_{\partial \omega}\left(\left.\frac{\partial\left(\mathcal{S}_{0} g\right)}{\partial \nu_{0}}\right|_{-}-\left.\frac{\partial\left(\mathcal{S}_{1} f\right)}{\partial \nu_{1}}\right|_{-}\right) \cdot h d \sigma_{y}
$$

where

$$
\frac{\partial h}{\partial \nu_{i}}:=2 \mu_{i} e(h) \nu_{y}
$$

Indeed, since $\varphi, \mathcal{S}_{1} f$ and $\mathcal{S}_{0} g$ are continuous across $\partial \omega$, we have from (42),

$$
\int_{\partial \omega} \varphi \cdot\left(\frac{\partial h}{\partial \nu_{1}}-\frac{\partial h}{\partial \nu_{0}}\right) d \sigma_{y}=\left.\int_{\partial \omega} \mathcal{S}_{1} f\right|_{-} \cdot \frac{\partial h}{\partial \nu_{1}} d \sigma_{y}-\left.\int_{\partial \omega} \mathcal{S}_{0} g\right|_{-} \cdot \frac{\partial h}{\partial \nu_{0}} d \sigma_{y} .
$$

Then, using the fact that $h$ is linear and divergence free, and that $\mathcal{S}_{0} g, \mathcal{S}_{1} f$ are, respectively, the solutions of the Stokes problem with viscosities $\mu_{0}$ and $\mu_{1}$, two integrations by parts yield (43).

In the sequel, using (42), we express the tensor $K$ in terms of layer potentials and simplify the resulting expression when $\omega$ satisfies the following geometric assumption:

Assumption. There exist numbers $c_{i j}, d_{i j}^{k l}, 1 \leq i, j, k, l \leq n$ such that for every $x \in \partial \omega$ and $i, j, k=1, \ldots, n$,

$$
\int_{\omega} \Gamma_{0}(x, y) \nu_{k}(y) d \sigma_{y}=\sum_{l=1}^{n} c_{k l} x_{l}
$$

and

$$
I_{k i j}(x)+I_{k j i}(x)=\sum_{l=1}^{n} d_{i j}^{k l} x_{l}
$$

where

$$
I_{k i j}(x):=\frac{1}{\omega_{n}} \int_{\partial \omega} \frac{\left(x_{k}-y_{k}\right)\left(x_{i}-y_{i}\right)}{|x-y|^{n}} \nu_{j}(y) d \sigma_{y}
$$


where $\omega_{n}$ is the area of the unit sphere in $\mathbb{R}^{n}$, and where $\Gamma_{0}$ denotes the fundamental solution of the Laplacian

$$
\forall x, y \in \mathbb{R}^{n}, x \neq y, \quad \Gamma_{0}(x, y) \quad:= \begin{cases}-\frac{1}{4 \pi} \frac{1}{|x-y|} & \text { if } n=3, \\ \frac{1}{2 \pi} \ln |x-y| & \text { if } n=2 .\end{cases}
$$

In other words, we asume that certain moments of $\Gamma_{0}(x, \cdot)$ computed on $\partial \omega$ are linear functions of $x$. It is verified in 4, 2, 18, 21 that ellipses in 2D and ellipsoids in 3D have this property. The converse is also true and was the main tool to prove the weak Eshelby conjecture [2, 18, 21].

Theorem 4.2. We set $h_{i j}:=y_{i} e^{j}-\frac{\delta_{i j}}{n} y$. Let $(f, g) \in L^{2}(\partial \omega)^{n} \times L^{2}(\partial \omega)^{n}$ be the solution to (41). Then, the curvature moment tensor $K$ defined by (18) satisfies

$$
K_{i j}=\tilde{\lambda} \int_{\partial \omega} h_{i j} \cdot g d \sigma_{y}+2 \tilde{\lambda} X_{i j}
$$

Moreover, under the assumptions (44) and (45), $K$ satisfies

$$
(\mathbf{I}-\mathbf{C}(\omega)) K-\frac{\tilde{\lambda} r}{n} \mathbf{C}(\omega) I_{n}=\tilde{\lambda}(\mathbf{I}-2 \mathbf{C}(\omega)) X,
$$

where $X$ is the symmetric matrix with zero trace given by (17), $I_{n} \in \mathbb{R}^{n \times n}$ is the identity matrix, $\mathbf{I}$ is the identity fourth-order tensor, $\mathbf{C}(\omega)$ is the symmetric fourth-order tensor of coefficients

$$
C(\omega)_{i j k l}=\frac{\mu_{1}-\mu_{0}}{4 \mu_{0}}\left(\delta_{k i} c_{j l}+\delta_{k j} c_{i l}+\delta_{l i} c_{j k}+\delta_{l j} c_{i k}-d_{i j}^{k l}-d_{i j}^{l k}\right),
$$

and the constant $r$ is given by

$$
r=\int_{\partial \omega} y \cdot g d \sigma_{y}
$$

REMARK 4.3.

(1) The tensor $\mathbf{C}(\omega)$ given by (50) is similar to the one considered in [7, but in our formula an additionnal term $\frac{r}{n} \mathbf{C}(\omega) I_{n}$ appears. This term comes from the fact that the tensor $K$ is expressed in terms of $h_{i j}=y_{i} e^{j}-\frac{\delta_{i j}}{n} y$ (i.e. in terms of a divergence-free field) instead of $y_{i} e^{j}$ in [7.

(2) In the cases of ellipses and ellipsoids, we will show that the additional term $\mathbf{C}(\omega) I_{n}$ is zero and hence $K$ can be computed from (49).

(3) As in [7, the system (49) is not necessarily invertible. In fact the tensor

$$
\widetilde{\mathbf{C}}(\omega)=\frac{\mu_{0}}{\mu_{1}-\mu_{0}} \mathbf{C}(\omega)
$$

only depends on the inclusion $\omega$ and so the system (49) is invertible for all the values of $\frac{\mu_{0}}{\mu_{1}-\mu_{0}} \in \mathbb{R}$ except the eigenvalues of $\widetilde{\mathbf{C}}(\omega)$. Later we will show some explicit eigenvalues of $\widetilde{\mathbf{C}}(\omega)$ in the case where $\omega$ is an ellipsoid. If $\frac{\mu_{0}}{\mu_{1}-\mu_{0}}$ belongs to the discrete set of eigenvalues of $\widetilde{\mathbf{C}}(\omega)$, the system (49) is no longer invertible and consequently the tensor $K$ cannot be retrieved directly from it. 
Proof. From (18), the curvature moment tensor is given, for $i, j=1, \ldots, n$, by

$$
\begin{aligned}
K_{i j} & =2 \tilde{\lambda}\left(\mu_{0}-\mu_{1}\right) \int_{\omega} e(\varphi)_{i j} d y+\tilde{\lambda} X_{i j} \\
& =2 \tilde{\lambda}\left(\mu_{0}-\mu_{1}\right) \int_{\omega} e(\varphi): e\left(h_{i j}\right) d y+\tilde{\lambda} X_{i j}
\end{aligned}
$$

where $\varphi$ is given by (42). Since $h_{i j}$ is linear and divergence free, we have from (43),

$$
\begin{aligned}
K_{i j}-\tilde{\lambda} X_{i j} & =\tilde{\lambda} \int_{\partial \omega} \varphi \cdot\left(\frac{\partial h_{i j}}{\partial \nu_{0}}-\frac{\partial h_{i j}}{\partial \nu_{1}}\right) d \sigma_{y} \\
& =\tilde{\lambda} \int_{\partial \omega}\left(\left.\frac{\partial\left(\mathcal{S}_{0} g\right)}{\partial \nu_{0}}\right|_{-}-\left.\frac{\partial\left(\mathcal{S}_{1} f\right)}{\partial \nu_{1}}\right|_{-}\right) \cdot h_{i j} d \sigma_{y} .
\end{aligned}
$$

From the second jump condition in (41) and from (40), we obtain

$$
\begin{aligned}
K_{i j}-\tilde{\lambda} X_{i j} & =\tilde{\lambda} \int_{\partial \omega}\left(\left.\frac{\partial\left(\mathcal{S}_{0} g\right)}{\partial \nu_{0}}\right|_{-}-\left.\frac{\partial\left(\mathcal{S}_{0} g\right)}{\partial \nu_{0}}\right|_{+}+\kappa \nu_{y}\right) \cdot h_{i j} d \sigma_{y} \\
& =\tilde{\lambda} \int_{\partial \omega} g \cdot h_{i j} d \sigma_{y}+\tilde{\lambda} \int_{\partial \omega} \kappa \nu_{y} \cdot h_{i j} d \sigma_{y},
\end{aligned}
$$

which, since $X_{i j}$ satisfies (19), yields (48). From (52) and the first jump condition in (41), we deduce

$$
\begin{aligned}
K_{i j}-\tilde{\lambda} X_{i j} & =\left.\tilde{\lambda} \int_{\partial \omega} \mathcal{S}_{1} f\right|_{-} \cdot \frac{\partial h_{i j}}{\partial \nu_{0}}-\left.\mathcal{S}_{0} g\right|_{-} \cdot \frac{\partial h_{i j}}{\partial \nu_{1}} d \sigma_{y} \\
& =\tilde{\lambda} \int_{\partial \omega}\left(\frac{\partial h_{i j}}{\partial \nu_{0}}-\frac{\partial h_{i j}}{\partial \nu_{1}}\right) \cdot \mathcal{S}_{0} g d \sigma_{y},
\end{aligned}
$$

and since $\mathcal{S}_{0}$ is a symmetric operator we obtain

$$
K_{i j}-\tilde{\lambda} X_{i j}=\tilde{\lambda} \int_{\partial \omega}\left(\mathcal{S}_{0} \frac{\partial h_{i j}}{\partial \nu_{0}}-\mathcal{S}_{0} \frac{\partial h_{i j}}{\partial \nu_{1}}\right) \cdot g d \sigma
$$

Next, using the assumptions (44) and (45) we express the above right-hand side in terms of $K$. Note that for $l=1, \ldots, n$,

$$
\begin{aligned}
\left(\frac{\partial h_{i j}}{\partial \nu_{0}}\right)_{l} & =2 \mu_{0}\left(e\left(h_{i j}\right) \nu_{y}\right)_{l}=2 \mu_{0} \sum_{m=1}^{n}\left(\frac{1}{2}\left(\delta_{i m} \delta_{j l}+\delta_{i l} \delta_{j m}\right)-\frac{1}{n} \delta_{i j} \delta_{l m}\right) \nu_{m} \\
& =\mu_{0}\left(\delta_{j l} \nu_{i}+\delta_{i l} \nu_{j}\right)-\frac{2 \mu_{0}}{n} \delta_{i j} \nu_{l} .
\end{aligned}
$$

Thus, we obtain that, for $k=1, \ldots, n$,

$$
\begin{aligned}
\mathcal{S}_{0}\left(\frac{\partial h_{i j}}{\partial \nu_{0}}\right)(x)_{k}= & \sum_{l=1}^{n} \frac{1}{\mu_{0}} \int_{\partial \omega} \Gamma_{k l}(x, y)\left(\frac{\partial h_{i j}}{\partial \nu_{0}}\right)_{l}(y) d \sigma_{y} \\
= & \int_{\partial \omega} \Gamma_{k j}(x, y) \nu_{i}(y) d \sigma_{y}+\int_{\partial \omega} \Gamma_{k i}(x, y) \nu_{j}(y) d \sigma_{y} \\
& -\frac{2}{n} \delta_{i j} \sum_{l=1}^{n} \int_{\partial \omega} \Gamma_{k l}(x, y) \nu_{l}(y) d \sigma_{y} .
\end{aligned}
$$


We further note that

$$
\sum_{l=1}^{n} \int_{\partial \omega} \Gamma_{k l}(x, y) \nu_{l}(y) d \sigma_{y}=\int_{\omega} \sum_{l=1}^{n} \frac{\partial \Gamma_{k l}}{\partial y_{l}}(x, y) d y=0,
$$

and, making explicit the relationship between the Green functions $\Gamma$ and $\Gamma_{0}$ as in [7] we arrive at

$$
\begin{aligned}
& \int_{\partial \omega} \Gamma_{k j}(x, y) \nu_{i}(y) d \sigma_{y}+\int_{\partial \omega} \Gamma_{k i}(x, y) \nu_{j}(y) d \sigma_{y} \\
& =-\frac{1}{2}\left(\delta_{k j} \int_{\partial \omega} \Gamma_{0}(x, y) \nu_{i}(y) d \sigma_{y}+\delta_{k i} \int_{\partial \omega} \Gamma_{0}(x, y) \nu_{j}(y) d \sigma_{y}-\left(I_{k i j}+I_{k j i}\right)\right),
\end{aligned}
$$

where $I_{k i j}$ is given by (46). From (44) and (45), we deduce

$$
\begin{aligned}
\mathcal{S}_{0}\left(\frac{\partial h_{i j}}{\partial \nu_{0}}\right)(x)_{k}=-\frac{1}{2}( & \delta_{k j} \int_{\partial \omega} \Gamma_{0}(x, y) \nu_{i}(y) d \sigma_{y} \\
& \left.\quad+\delta_{k i} \int_{\partial \omega} \Gamma_{0}(x, y) \nu_{j}(y) d \sigma_{y}-\left(I_{k i j}+I_{k j i}\right)\right) \\
= & -\frac{1}{2} \sum_{l=1}^{n}\left(\delta_{k j} c_{i l}+\delta_{k i} c_{j l}-d_{i j}^{k l}\right) x_{l} .
\end{aligned}
$$

Proceeding similarly for the term $\mathcal{S}_{0}\left(\frac{\partial h_{i j}}{\partial \nu_{1}}\right)$ yields

$$
\mathcal{S}_{0}\left(\frac{\partial h_{i j}}{\partial \nu_{1}}\right)(x)_{k}=-\frac{\mu_{1}}{2 \mu_{0}} \sum_{l=1}^{n}\left(\delta_{k j} c_{i l}+\delta_{k i} c_{j l}-d_{i j}^{k l}\right) x_{l} .
$$

It follows that

$$
\begin{aligned}
\tilde{\lambda} & \int_{\partial \omega}\left(\mathcal{S}_{0}\left(\frac{\partial h_{i j}}{\partial \nu_{0}}\right)-\mathcal{S}_{0}\left(\frac{\partial h_{i j}}{\partial \nu_{1}}\right)\right) \cdot g d \sigma_{y} \\
= & \tilde{\lambda} \sum_{k, l=1}^{n} \frac{\mu_{1}-\mu_{0}}{2 \mu_{0}}\left(\delta_{k j} c_{i l}+\delta_{k i} c_{j l}-d_{i j}^{k l}\right) \int_{\partial \omega} y_{l} e^{k} \cdot g d \sigma_{y} \\
= & \sum_{k, l=1}^{n} \frac{\mu_{1}-\mu_{0}}{2 \mu_{0}}\left(\delta_{k j} c_{i l}+\delta_{k i} c_{j l}-d_{i j}^{k l}\right)\left(K_{l k}-2 X_{l k}\right) \\
& +\frac{\tilde{\lambda}}{n} \sum_{k, l=1}^{n} \frac{\mu_{1}-\mu_{0}}{2 \mu_{0}}\left(\delta_{k j} c_{i l}+\delta_{k i} c_{j l}-d_{i j}^{k l}\right) \delta_{k l} \int_{\partial \omega} y \cdot g d \sigma_{y} .
\end{aligned}
$$

Since $K$ is symmetric, the previous equality can be rewritten as

$$
\begin{aligned}
& \tilde{\lambda} \int_{\partial \omega}\left(\mathcal{S}_{0}\left(\frac{\partial h_{i j}}{\partial \nu_{0}}\right)-\mathcal{S}_{0}\left(\frac{\partial h_{i j}}{\partial \nu_{1}}\right)\right) \cdot g d \sigma_{y} \\
&=\sum_{k, l=1}^{n} C(\omega)_{i j k l}\left(K_{l k}-2 \tilde{\lambda} X_{l k}\right)+\frac{\tilde{\lambda}}{n} \sum_{k=1}^{n} C(\omega)_{i j k k} \int_{\partial \omega} y \cdot g d \sigma_{x} \\
&=(\mathbf{C}(\omega) K)_{i j}-2 \tilde{\lambda}(\mathbf{C}(\omega) X)_{i j}+\frac{\tilde{\lambda} r}{n}(\mathbf{C}(\omega) I)_{i j}
\end{aligned}
$$


where $C(\omega)_{i j k l}$ and $r$ are given by (50) and (51) respectively, and (49) follows from (55) and (56).

4.1. The curvature moment tensor of an ellipse. We first recall the expression of the viscous moment tensor $V$ obtained in 4 .

Proposition 4.4 (Ammari et al. [4]). Suppose that $\omega$ is the ellipse defined by

$$
\frac{x_{1}^{2}}{a^{2}}+\frac{x_{2}^{2}}{b^{2}}=1, \quad \text { where } \quad a \geq b>0 .
$$

Then, the nonzero coefficients of its viscous moment tensor $V$ are

$$
\left\{\begin{array}{l}
V_{1111}=V_{2222}=-V_{1122}=-V_{2211}=\frac{2 \mu_{0}\left(\mu_{1}-\mu_{0}\right)(a+b)^{2}|\omega|}{\left(\mu_{0}+\mu_{1}\right)(a+b)^{2}+\left(\mu_{0}-\mu_{1}\right)(a-b)^{2}}, \\
V_{1212}=V_{2112}=V_{1221}=V_{2121}=\frac{2 \mu_{0}\left(\mu_{1}-\mu_{0}\right)(a+b)^{2}|\omega|}{\left(\mu_{0}+\mu_{1}\right)(a+b)^{2}+\left(\mu_{1}-\mu_{0}\right)(a-b)^{2}} .
\end{array}\right.
$$

For the curvature moment tensor, we have the following result.

Proposition 4.5. Suppose that $\omega$ is the ellipse defined by (57). Then, its curvature moment tensor is

$$
K(a, b)=2 \tilde{\lambda} \frac{\mu_{1}(a+b)^{2}+\left(\mu_{0}-\mu_{1}\right)(a-b)^{2}}{\left(\mu_{0}+\mu_{1}\right)(a+b)^{2}+\left(\mu_{0}-\mu_{1}\right)(a-b)^{2}} \chi(a, b)\left(\begin{array}{cc}
1 & 0 \\
0 & -1
\end{array}\right),
$$

where $\chi(a, b) \geq 0$ is given by

$$
\chi(a, b):=\int_{0}^{2 \pi} \frac{a^{2} \sin ^{2} \theta-b^{2} \cos ^{2} \theta}{\sqrt{a^{2} \sin ^{2} \theta+b^{2} \cos ^{2} \theta}} d \theta .
$$

REMARK 4.6.

(1) We note that the denominator in (59) can be rewritten as

$$
\left(\mu_{0}+\mu_{1}\right)(a+b)^{2}+\left(\mu_{0}-\mu_{1}\right)(a-b)^{2}=\left(\mu_{0}-\mu_{1}\right)(a+b)^{2}\left(\frac{\mu_{0}+\mu_{1}}{\mu_{0}-\mu_{1}}+\left(\frac{a-b}{a+b}\right)^{2}\right) \text {. }
$$

Since $a, b, \mu_{0}, \mu_{1}$ are strictly positive constants, we have

$$
\left|\frac{\mu_{0}+\mu_{1}}{\mu_{0}-\mu_{1}}\right|>1, \quad 0 \leq\left(\frac{a-b}{a+b}\right)^{2}<1,
$$

and thus, the denominator is always different from zero.

(2) In the case where $a=b$, we have $\chi(a, b)=0$ so that $K=0$, as already noted in Remark 2.3.

(3) If $|\omega|$ is fixed, one can express $K$ as a function of the eccentricity $e=\sqrt{1-\frac{b^{2}}{a^{2}}} \epsilon$ $[0,1[$. For example, if $|\omega|=\pi$ (i.e. $a b=1$ ), from (59), we have

$$
K=\zeta(e)\left(\begin{array}{cc}
1 & 0 \\
0 & -1
\end{array}\right),
$$

where

$$
\zeta(e)=2 \tilde{\lambda} \frac{\mu_{1}\left(1+\sqrt{1-e^{2}}\right)^{2}+\left(\mu_{0}-\mu_{1}\right)\left(1-\sqrt{1-e^{2}}\right)^{2}}{\left(\mu_{0}+\mu_{1}\right)\left(1+\sqrt{1-e^{2}}\right)^{2}+\left(\mu_{0}-\mu_{1}\right)\left(1-\sqrt{1-e^{2}}\right)^{2}} \chi\left(\left(1-e^{2}\right)^{-1 / 4},\left(1-e^{2}\right)^{1 / 4}\right) .
$$


The graph of the real-valued function $\zeta(e)$ for the particular case where $\tilde{\lambda}=$ $1, \mu_{0}=1$ and $\mu_{1}=2$ is shown in Figure 1 .

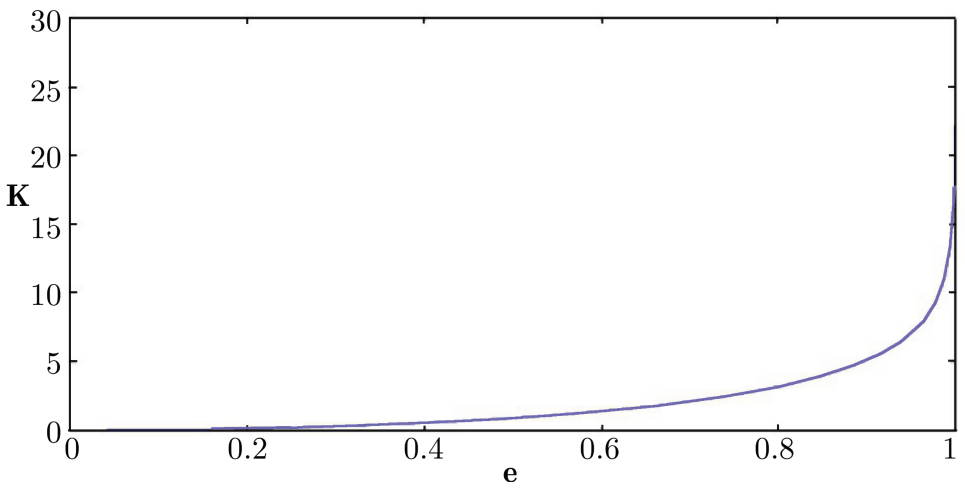

FIG. 1. Representation of $K$ as a function of the eccentricity $e$ for $\left(\mu_{0}, \mu_{1}\right)=(1,2)$ and $\tilde{\lambda}=1$.

Proof. From (17), using the elliptical coordinates $x_{1}=a \cos \theta, x_{2}=b \sin \theta$ with $\theta \in$ $[0,2 \pi)$, we have

$$
X_{12}=X_{21}=-\int_{\partial \omega} \nu_{1} \nu_{2} d \sigma=-\int_{0}^{2 \pi} \frac{a b \cos \theta \sin \theta}{\sqrt{a^{2} \sin ^{2} \theta+b^{2} \cos ^{2} \theta}} d \theta=0
$$

and

$$
\begin{aligned}
X_{11} & =-\int_{0}^{2 \pi} \frac{b^{2} \cos ^{2} \theta}{\sqrt{a^{2} \sin ^{2} \theta+b^{2} \cos ^{2} \theta}} d \theta+\frac{1}{2} \int_{0}^{2 \pi} \sqrt{a^{2} \sin ^{2} \theta+b^{2} \cos ^{2} \theta} d \theta \\
& =-\frac{1}{2} \int_{0}^{2 \pi} \sqrt{a^{2} \sin ^{2} \theta+b^{2} \cos ^{2} \theta} d \theta+\int_{0}^{2 \pi} \frac{a^{2} \sin ^{2} \theta}{\sqrt{a^{2} \sin ^{2} \theta+b^{2} \cos ^{2} \theta}} d \theta \\
& =-X_{22}=\frac{1}{2} \chi(a, b) .
\end{aligned}
$$

We set $m:=\frac{a-b}{a+b}$. We know from [7, section 3, that assumptions (44) and (45) are satisfied by $\omega$. In addition, it is shown there that

$$
\forall k, l=1,2, \quad c_{k l}=\delta_{k l} s_{k}, \quad \text { where } \quad s_{1}=-\frac{1}{2}(1-m) \quad \text { and } \quad s_{2}=-\frac{1}{2}(1+m),
$$

and

$$
\begin{aligned}
& d_{11}^{11}=m+s_{3}, d_{11}^{22}=-\left(1+s_{3}\right), d_{12}^{12}=s_{1}-s_{3}, d_{12}^{21}=s_{2}-s_{3}, d_{22}^{11}=-\left(1+s_{3}\right), d_{22}^{22}= \\
& -m+s_{3}, d_{11}^{12}=d_{11}^{21}=d_{12}^{11}=d_{12}^{22}=d_{21}^{11}=d_{21}^{22}=d_{22}^{12}=d_{22}^{21}=0 \text { with } s_{3}=-\frac{1}{2}\left(1+m^{2}\right) .
\end{aligned}
$$

In the sequel, we identify the fourth-order tensors in dimension 2 with $3 \times 3$ matrices using the conventional identification

$$
(11) \rightarrow 1,(22) \rightarrow 2,(12) \rightarrow 3,
$$


and the $2 \times 2$ symmetric matrices are identified with vectors of $\mathbb{R}^{3}$. The tensor $\mathbf{C}(\omega)$ can be written as the $3 \times 3$ matrix

$$
\mathbf{C}(\omega)=\frac{\mu_{1}-\mu_{0}}{4 \mu_{0}}\left(\begin{array}{ccc}
m^{2}-1 & 1-m^{2} & 0 \\
1-m^{2} & m^{2}-1 & 0 \\
0 & 0 & -2\left(m^{2}+1\right)
\end{array}\right) .
$$

Using the identification (63), we deduce from (64) that

$$
\mathbf{C}(\omega) I=0 .
$$

Set

$$
\mathbf{A}:=\mathbf{I}-\mathbf{C}(\omega)=\left(\begin{array}{ccc}
\alpha & \beta & 0 \\
\beta & \alpha & 0 \\
0 & 0 & \gamma
\end{array}\right)
$$

where

$$
\alpha=\frac{3 \mu_{0}+\mu_{1}+\left(\mu_{0}-\mu_{1}\right) m^{2}}{4 \mu_{0}}, \beta=\frac{\mu_{0}-\mu_{1}}{4 \mu_{0}}\left(1-m^{2}\right) \text { and } \gamma=\frac{\mu_{1}+\mu_{0}+\left(\mu_{1}-\mu_{0}\right) m^{2}}{2 \mu_{0}} .
$$

Note that $\alpha+\beta=1$, and so we have

$$
\operatorname{det}(\mathbf{A})=\left(\alpha^{2}-\beta^{2}\right) \gamma=(\alpha-\beta) \gamma \neq 0,
$$

In fact, $\alpha-\beta=0$, or $\gamma=0$, combined with $m \leq 1$ implies $\mu_{0} \leq 0$ or $\mu_{1} \leq 0$. Consequently, A is invertible and

$$
\mathbf{A}^{-1}=\frac{1}{\alpha-\beta}\left(\begin{array}{ccc}
\alpha & -\beta & 0 \\
-\beta & \alpha & 0 \\
0 & 0 & 0
\end{array}\right)+\frac{1}{\gamma}\left(\begin{array}{lll}
0 & 0 & 0 \\
0 & 0 & 0 \\
0 & 0 & 1
\end{array}\right) .
$$

Moreover, since $X_{12}=X_{21}=0$ and $X_{11}=-X_{22}$, the matrix $X$ can be written as

$$
X=X_{11}\left(\begin{array}{c}
1 \\
-1 \\
0
\end{array}\right) \text {. }
$$

With the identification (63), we have

$$
\mathbf{I}-2 \mathbf{C}(\omega)=\left(\begin{array}{ccc}
\alpha^{\prime} & \beta^{\prime} & 0 \\
\beta^{\prime} & \alpha^{\prime} & 0 \\
0 & 0 & \gamma^{\prime}
\end{array}\right)
$$

where

$\alpha^{\prime}=\frac{\mu_{0}+\mu_{1}+\left(\mu_{0}-\mu_{1}\right) m^{2}}{2 \mu_{0}}, \beta^{\prime}=\frac{\mu_{0}-\mu_{1}}{2 \mu_{0}}\left(1-m^{2}\right)$ and $\gamma^{\prime}=\frac{2 \mu_{0}-\mu_{1}+\left(\mu_{1}-\mu_{0}\right) m^{2}}{\mu_{0}}$.

It follows from (65) that

$$
K=\tilde{\lambda} \mathbf{A}^{-1}(\mathbf{I}-2 \mathbf{C}(\omega)) X=\tilde{\lambda}\left(\alpha^{\prime}-\beta^{\prime}\right) \mathbf{A}^{-1} X=\tilde{\lambda} \frac{\left(\alpha^{\prime}-\beta^{\prime}\right)}{\alpha-\beta} X .
$$

Thus, from (61) and (62), we obtain (59). 
4.2. Curvature moment tensor for ellipsoids. In this section, we consider the case where $\omega$ is an ellipsoid of the form

$$
\frac{x_{1}^{2}}{c_{1}^{2}}+\frac{x_{2}^{2}}{c_{2}^{2}}+\frac{x_{3}^{2}}{c_{3}^{2}}=1, \quad \text { where } \quad c_{i}>0, \forall i=1,2,3 .
$$

We show that $K$ is a diagonal tensor and can be derived explicitly by solving a linear system (similar to the case of an ellipse in dimension 2). For the sake of brevity we only give the coefficients of the linear system. For $i, j=1,2,3, i \neq j$, we set

$$
\left\{\begin{aligned}
s_{i} & =-\frac{c_{1} c_{2} c_{3}}{2} \int_{0}^{+\infty} \frac{d s}{\left(c_{i}^{2}+s\right) \sqrt{g(s)}} \\
s_{i j} & =-\frac{c_{1} c_{2} c_{3}\left(c_{i}^{2}+c_{j}^{2}\right)}{2} \int_{0}^{+\infty} \frac{d s}{\left(c_{i}^{2}+s\right)\left(c_{j}^{2}+s\right) \sqrt{g(s)}} \\
g(s) & =\left(c_{1}^{2}+s\right)\left(c_{2}^{2}+s\right)\left(c_{3}^{2}+s\right)
\end{aligned}\right.
$$

REMARK 4.7. Note that, for $i, j=1,2,3, i \neq j, s_{i}<0$ and $s_{i j}<0$ since $c_{i}>0$.

Theorem 4.8. Suppose that $\omega$ is an ellipsoid of the form (66). Then, the curvature moment tensor $K$ is diagonal. If in addition

$$
\frac{\mu_{0}}{\mu_{1}-\mu_{0}} \neq s_{i j} \quad \text { for } \quad i, j=1,2,3
$$

then the vector of diagonal coefficients $K_{s}:=\left(K_{i i}\right) \in \mathbb{R}^{3}$ satisfies

$$
\left(I-C_{s}\right) K_{s}=\tilde{\lambda}\left(I-2 C_{s}\right) X_{s},
$$

where $C_{s} \in \mathbb{R}_{s}^{3 \times 3}$ is given by

$C_{s}=\frac{\mu_{1}-\mu_{0}}{2 \mu_{0}}\left(\begin{array}{ccc}2 s_{1}+s_{2}+s_{3}-s_{12}-s_{13} & s_{12}-s_{1}-s_{2} & s_{13}-s_{1}-s_{3} \\ s_{12}-s_{1}-s_{2} & 2 s_{2}+s_{1}+s_{3}-s_{21}-s_{23} & s_{23}-s_{2}-s_{3} \\ s_{13}-s_{1}-s_{3} & s_{23}-s_{2}-s_{3} & 2 s_{3}+s_{1}+s_{2}-s_{31}-s_{32}\end{array}\right)$,

with $s_{i}, s_{i j}$ defined by (67) and $X_{s}=-\left(\chi_{1}, \chi_{2}, \chi_{3}\right)^{T} \in \mathbb{R}^{3}$ with

$$
\left\{\begin{array}{l}
\chi_{1}=\int_{0}^{\pi} \int_{0}^{2 \pi} \frac{c_{2}^{2} c_{3}^{2} \cos ^{2} \theta \sin ^{3} \psi}{h(\theta, \psi)} d \psi d \theta-\frac{1}{3} \int_{0}^{\pi} \int_{0}^{2 \pi} h(\theta, \psi) \sin \psi d \psi d \theta, \\
\chi_{2}=\int_{0}^{\pi} \int_{0}^{2 \pi} \frac{c_{1}^{2} c_{3}^{2} \sin ^{2} \theta \sin ^{3} \psi}{h(\theta, \psi)} d \psi d \theta-\frac{1}{3} \int_{0}^{\pi} \int_{0}^{2 \pi} h(\theta, \psi) \sin \psi d \psi d \theta \\
\chi_{3}=\int_{0}^{\pi} \int_{0}^{2 \pi} \frac{c_{1}^{2} c_{2}^{2} \cos ^{2} \psi \sin \psi}{h(\theta, \psi)} d \psi d \theta-\frac{1}{3} \int_{0}^{\pi} \int_{0}^{2 \pi} h(\theta, \psi) \sin \psi d \psi d \theta
\end{array}\right.
$$

where

$$
h(\theta, \psi):=\sqrt{c_{1}^{2} c_{2}^{2} \cos ^{2} \psi+c_{2}^{2} c_{3}^{2} \cos ^{2} \theta \sin ^{2} \psi+c_{1}^{2} c_{3}^{2} \sin ^{2} \theta \sin ^{2} \psi}
$$

REMARK 4.9.

(1) The assumption (68) is fulfilled if $\mu_{1}>\mu_{0}$. Indeed, if there exists $i, j \in\{1,2,3\}$ such that (68) is not satisfied, then Remark 4.7 implies that

$$
\mu_{0}=s_{i j}\left(\mu_{1}-\mu_{0}\right)<0,
$$

which is in contradiction with $\mu_{0}>0$. 
(2) The constants $s_{i j}, i, j \in\{1,2,3\}$ only depend on the parameters of the ellipsoid and belong to the set of eigenvalues of the tensor $\widetilde{\mathbf{C}}(\omega)$ defined in Remark 4.3 .

(3) Assume that $\omega$ is a ball. Then condition (68) is satisfied, $I-C_{s}$ is invertible and $X_{s}=0$. Indeed, if $c_{1}=c_{2}=c_{3}$, simple computations show that $X_{s}=$ $0, s_{i}=-1 / 3$ and $s_{i j}=-2 / 5$ for every $i, j=1,2,3$. If (68) were not satisfied for some $i, j \in\{1,2,3\}$, one would obtain $3 \mu_{0}=-2 \mu_{1}$, a contradiction as both viscosities are positive. Equality (69) implies then that $K=0$, in accordance with Remark 2.3 .

Proof. In order to compute $X$, we introduce the spherical coordinates $x_{1}=$ $c_{1} \cos \theta \sin \psi, x_{2}=c_{2} \sin \theta \sin \psi, x_{3}=c_{3} \cos \psi$ with $\psi \in[0, \pi]$ and $\theta \in[0,2 \pi]$. We obtain

$$
\nu=\frac{1}{h(\theta, \psi)}\left(\begin{array}{c}
c_{2} c_{3} \cos \theta \sin \psi \\
c_{1} c_{3} \sin \theta \sin \psi \\
c_{1} c_{2} \cos \psi
\end{array}\right)=\frac{1}{h(\theta, \psi)}\left(\begin{array}{c}
\alpha_{1} \\
\alpha_{2} \\
\alpha_{3}
\end{array}\right),
$$

where $h(\theta, \psi)$ is given by (72). From (17), we have

$$
X_{i j}=-\int_{0}^{\pi} \int_{0}^{2 \pi} \frac{\alpha_{i} \alpha_{j} \sin \psi}{h(\theta, \psi)} d \psi d \theta+\frac{\delta_{i j}}{3} \int_{0}^{\pi} \int_{0}^{2 \pi} h(\theta, \psi) \sin \psi d \psi d \theta .
$$

Simple computations show that $X_{i j}=0$ for $i \neq j$ and $X_{i i}=\chi_{i}$. It is shown in [7] that assumptions (44) and (45) are satisfied, with

$$
c_{i j}=s_{i} \delta_{i j}, \quad \forall i, j \in\{1,2,3\},
$$

and where the coefficients $d_{i j}^{k l}$ are

$$
\left\{\begin{array}{l}
d_{i j}^{i j}=s_{i}-s_{i j}, \quad d_{i j}^{j i}=s_{j}-s_{i j}, \\
d_{i i}^{j j}=s_{i}+s_{j}-s_{i j}, \quad d_{i i}^{i i}=-\sum_{k \neq i} s_{k}+\sum_{k \neq i} s_{i k},
\end{array}\right.
$$

for $i \neq j$, and are zero otherwise. Recalling (50), we have for $1 \leq i, j \leq 3$,

$$
(C(\omega) I)_{i j}=\frac{\mu_{1}-\mu_{0}}{2 \mu_{0}}\left(2 \delta_{i j} s_{i}-\sum_{k=1}^{3} d_{i j}^{k k}\right) .
$$

If $i \neq j$, we easily obtain $(C(\omega) I)_{i j}=0$. For $i=j$, we have

$$
\begin{aligned}
(C(\omega) I)_{i j} & =\frac{\mu_{1}-\mu_{0}}{2 \mu_{0}}\left(2 s_{i}+\sum_{k \neq i} s_{k}-\sum_{k \neq i} s_{i k}-\sum_{k \neq i} d_{i i}^{k k}\right) \\
& =\frac{\mu_{1}-\mu_{0}}{2 \mu_{0}}\left(2 s_{i}+\sum_{k \neq i} s_{k}-\sum_{k \neq i} s_{i k}-2 s_{i}-\sum_{k \neq i}\left(s_{k}+s_{i k}\right)\right)=0 .
\end{aligned}
$$

It follows from (49) that

$$
(\mathbf{I}-\mathbf{C}(\omega)) K=\tilde{\lambda}(\mathbf{I}-2 \mathbf{C}(\omega)) X
$$


Moreover, the definition (50) of $C(\omega)$ shows that, if 3 of the indices $i, j, k, l$ are distinct, then $C(\omega)_{i j k l}=0$. If $k=i, l=j$, we obtain

$$
C(\omega)_{i j k l}=\frac{\mu_{1}-\mu_{0}}{4 \mu_{0}}\left(s_{j}+s_{i}-\left(s_{i}-s_{i j}\right)-\left(s_{j}-s_{i j}\right)\right)=\frac{\mu_{1}-\mu_{0}}{2 \mu_{0}} s_{i j} .
$$

If $i \neq j$, equality (73) yields

$$
\left(1-\frac{\left(\mu_{1}-\mu_{0}\right) s_{i j}}{\mu_{0}}\right) K_{i j}=\tilde{\lambda}\left(1-\frac{2\left(\mu_{1}-\mu_{0}\right) s_{i j}}{\mu_{0}}\right) X_{i j}=0 .
$$

If (68) is satisfied, then $K_{i j}=0$ for $i \neq j$. We note that $K$ is defined as the moments of the function $\varphi$ solution to (12). Hence, it depends continuously on the coefficients of the PDE. In other words, $K$ is a continuous function of the contrast $\mu_{1} / \mu_{0}$. Since (68) only involves 3 values of the contrast, the continuity of $K$ shows that it must be diagonal for all choices of $\mu_{0}$ and $\mu_{1}$.

Finally, for $i=j$, we obtain from (74) that

$$
(\mathbf{C}(\omega) K)_{i i}=\frac{\mu_{1}-\mu_{0}}{2 \mu_{0}} \sum_{k=1}^{3}\left(\delta_{k i} s_{i}-d_{i i}^{k k}\right) K_{k k}=\left(C_{s} K_{s}\right)_{i},
$$

where $K_{s}=\left(K_{11}, K_{22}, K_{33}\right)^{T} \in \mathbb{R}^{3}$, and $C_{s}$ is given by (70), and (69) follows.

Appendix A. Integration by parts on a surface. We recall the following classical result of differential calculus (see e.g. [15]).

Proposition A.1. Let $\Omega$ be a bounded open subset of $\mathbb{R}^{n}$ with $W^{2, \infty}$ boundary. Let $f \in$ $W^{2,1}(\Omega)$ and $u \in C^{1}\left(\mathbb{R}^{n}\right)^{n}$ be uniformly bounded. Then, we have the following integration by parts formula on $\partial \Omega$ :

$$
\int_{\partial \Omega} u \cdot \nabla f+f \operatorname{div}_{\partial \Omega}(u) d \sigma=\int_{\partial \Omega}\left(\frac{\partial f}{\partial \nu}+\kappa f\right) u \cdot \nu d \sigma
$$

where $\nu$ is the outward unit normal to $\partial \Omega, \kappa$ is the mean curvature of $\partial \Omega$ and $\operatorname{div}_{\partial \Omega}(u)$ is defined by

$$
\operatorname{div}_{\partial \Omega}(u):=\operatorname{div}(u)-D u \nu \cdot \nu
$$

In particular, if $u$ and $f$ are constants, formula (75) reduces to

$$
\int_{\partial \Omega} f \kappa \nu \cdot u d \sigma=0
$$

Appendix B. Well-posedness of the Stokes system in $\mathbb{R}^{n}$. Recall that $\omega$ is a $C^{2}$ domain, and introduce the following spaces:

$$
\begin{aligned}
H_{0}^{-\frac{1}{2}}(\partial \omega)^{n} & :=\left\{g \in H^{-\frac{1}{2}}(\partial \omega)^{n} \mid \int_{\partial \omega} g \cdot \nu_{y} d \sigma_{y}=0\right\} \\
H_{0}^{\frac{1}{2}}(\partial \omega)^{n} & :=\left\{g \in H^{\frac{1}{2}}(\partial \omega)^{n} \mid \int_{\partial \omega} g \cdot \nu_{y} d \sigma_{y}=0\right\}
\end{aligned}
$$


where $H^{-\frac{1}{2}}(\partial \omega)=\left(H^{\frac{1}{2}}(\partial \omega)\right)^{\prime}$.

Let $f(x)$ be a vector function in $H^{-\frac{1}{2}}(\partial \omega)^{n}$ satisfying $\int_{\partial \omega} f d \sigma_{y}=0$. We consider the Stokes system

$$
\left\{\begin{aligned}
-\operatorname{Div}(2 \mu e(u))+\nabla p & =f \chi_{\partial \omega} & & \text { in } \mathbb{R}^{n} \\
\operatorname{div}(u) & =0 & & \text { in } \mathbb{R}^{n}
\end{aligned}\right.
$$

where $\mu$ is given by (10). In the rest of this section, we first recall some results of potential theory, then show that the above Stokes system is well-posed with the radiation conditions

$$
\left\{\begin{array}{rlll}
u(x)=O\left(|x|^{-1}\right) & \text { as } & |x| \rightarrow \infty \\
e(u)(x)=O\left(|x|^{-2}\right) & \text { as } & |x| \rightarrow \infty \\
p(x)=O\left(|x|^{-2}\right) & \text { as } & |x| \rightarrow \infty .
\end{array}\right.
$$

B.1. Potential theory for the Stokes system. The fundamental solution $(\boldsymbol{\Gamma}, P)$ of the Stokes system in $\mathbb{R}^{n}$ is given by (36) for $n=3$, and by (37) for $n=2$. We recall that the $i$ th row, $\Gamma_{i}$ of $\boldsymbol{\Gamma}$, satisfies

$$
\left\{\begin{aligned}
-\Delta_{y} \Gamma_{i}(y, z)+\nabla_{y} P_{i}(y, z) & =e^{i} \delta_{z}(y) & & \text { in } \mathbb{R}^{n} \\
\operatorname{div}_{y}\left(\Gamma_{i}\right)(y, z) & =0 & & \text { in } \mathbb{R}^{n}
\end{aligned}\right.
$$

The hydrodynamic single layer potential with density $\psi \in L^{2}(\partial \omega)^{n}$ is the vector function $\mathcal{S} \psi(x)$ whose components are defined by

$$
\mathcal{S}_{k} \psi(x):=\int_{\partial \omega} \Gamma_{k}(x, y) \cdot \psi(y) d \sigma_{y}, \quad x \in \mathbb{R}^{n} \backslash \partial \omega, \quad k=1, \ldots, n .
$$

The hydrodynamic double layer potential with density $\varphi \in L^{2}(\partial \omega)^{n}$ is the vector function $\mathcal{D} \varphi(x)$ whose components are given by

$$
\begin{aligned}
\mathcal{D}_{k} \varphi(x):=\int_{\partial \omega}\left(2 e_{y}\left(\Gamma_{k}\right)(x, y)-P_{k}(x, y) I_{n}\right) \nu_{y} \cdot \varphi(y) & d \sigma_{y}, \\
x & \in \mathbb{R}^{n} \backslash \partial \omega, \quad k=1, \ldots, n .
\end{aligned}
$$

In addition, the corresponding pressures $\mathcal{Q}$ and $\Pi$ are respectively

$$
\begin{aligned}
\mathcal{Q} \psi(x) & :=\int_{\partial \omega} P(x, y) \cdot \psi(y) d \sigma_{y} \quad x \in \mathbb{R}^{n} \backslash \partial \omega, \\
\Pi \varphi(x) & :=-2 \int_{\partial \omega} \nabla_{y} P(x, y) \nu_{y} \cdot \varphi(y) d \sigma_{y} \quad x \in \mathbb{R}^{n} \backslash \partial \omega .
\end{aligned}
$$

The pairs $(\mathcal{S} \psi, \mathcal{Q} \psi)$ and $(\mathcal{D} \varphi, \Pi \varphi)$ are classical solutions to the Stokes system in both regions $\omega$ and $\mathbb{R}^{n} \backslash \bar{\omega}$. For $\varphi \in L^{2}(\partial \omega)^{n}$, the following trace relations for $\mathcal{D}$ and $\mathcal{S}$ hold [19]:

$$
\begin{cases}\left.\mathcal{S} \varphi\right|_{+}=\left.\mathcal{S} \varphi\right|_{-} & \text {a.e. on } \partial \omega \\ \left.\mathcal{D} \varphi\right|_{ \pm}=\left( \pm \frac{1}{2} I+\mathcal{K}\right) \varphi & \text { a.e. on } \partial \omega\end{cases}
$$


where $\mathcal{K}$ is defined by

$$
\begin{aligned}
\mathcal{K} \varphi(x) \cdot e^{k}:=\text { p.v. } \int_{\partial \omega}\left(2 e_{y}\left(\Gamma_{k}\right)(x, y)+P_{k}(x, y) I_{n}\right) \nu_{y} \cdot \varphi(y) d \sigma_{y}, & \\
x & \in \partial \omega, \quad k=1, \ldots, n .
\end{aligned}
$$

The following result holds [3, 19]:

Lemma B.1. $\quad$ i. $\mathcal{S}: H_{0}^{-\frac{1}{2}}(\partial \omega) \rightarrow H_{0}^{\frac{1}{2}}(\partial \omega)$ is invertible.

ii. $\sigma I+\mathcal{K}: H^{-\frac{1}{2}}(\partial \omega) \rightarrow H^{\frac{1}{2}}(\partial \omega)$ is invertible for $|\sigma|>\frac{1}{2}$.

Let $(u, p)$ denote a solution to (78/79) which is smooth in $\omega$ and in $\mathcal{R}^{n} \backslash \omega$. Applying the Green formula inside $\omega$ yields

$$
2 \mu_{1} u(x)=S\left(\left.\frac{\partial u}{\partial \nu_{1}}\right|_{-}\right)(x)-2 \mu_{1} \mathcal{D} u(x), \quad \forall x \in \omega,
$$

where the conormal derivative $\partial / \partial \nu_{i}$ is defined by (39). Using the trace relations for $\mathcal{D}$ and $\mathcal{S}$ on $\partial \omega$, we obtain

$$
2 \mu_{1}\left(\frac{1}{2} I+\mathcal{K}\right) u(x)=\mathcal{S}\left(\left.\frac{\partial u}{\partial \nu_{1}}\right|_{-}\right)(x), \quad \forall x \in \omega .
$$

Let $R>0$ be large enough so that $\bar{\omega} \subset B_{R}(0)$. Multiplying the equations (78) by $\boldsymbol{\Gamma}$ and integrating by parts over $B_{R} \backslash \bar{\omega}$, we find

$$
2 \mu_{0} u(x)=-\mathcal{S}\left(\left.\frac{\partial u}{\partial \nu_{0}}\right|_{+}\right)(x)+2 \mu_{0} \mathcal{D} u(x)+I_{R}(x), \quad \forall x \in B_{R} \backslash \bar{\omega},
$$

where

$$
I_{R}(x) \cdot e_{i}=\int_{\partial B_{R}}\left(\left.\Gamma_{i} \cdot\left(2 \mu_{0} e(u)-p I_{n}\right) \frac{y}{R}\right|_{+}-\left.2 \mu_{0}\left(e_{y}\left(\Gamma_{i}\right)-P_{i} I_{n}\right) \frac{y}{R}\right|_{+} \cdot u\right) d \sigma_{y} .
$$

It follows from (79) that $I_{R} \rightarrow 0$ as $R \rightarrow \infty$. Therefore

$$
\mu_{0} u(x)=-\mathcal{S}\left(\left.\frac{\partial u}{\partial \nu_{0}}\right|_{+}\right)(x)+\mu_{0} \mathcal{D} u(x), \quad \forall x \in \mathbb{R}^{n} \backslash \bar{\omega} .
$$

When $x \rightarrow \partial \omega$ we obtain

$$
\mu_{0}\left(-\frac{1}{2} I+\mathcal{K}\right) u(x)=\mathcal{S}\left(\left.\frac{\partial u}{\partial \nu_{0}}\right|_{+}\right)(x), \quad \forall x \in \partial \omega .
$$

We recall that, since $\boldsymbol{\Gamma}$ is divergence free, we have $\mathcal{S} \nu=0$. Now, combining both equations (86) and (87) and using the jump $\left.\frac{\partial u}{\partial \nu_{1}}\right|_{-}-\left.\frac{\partial u}{\partial \nu_{0}}\right|_{+}=f$, proves the following.

Proposition B.2. If $(u, p)$ is a solution of (78)-(79), smooth in $\omega$ and in $\mathbb{R}^{n} \backslash \omega$, then $u$ satisfies the integral equation

$$
\left(\sigma_{0} I-\mathcal{K}\right) u(x)=\frac{1}{\left(\mu_{1}-\mu_{0}\right)} \mathcal{S} f(x), \quad \forall x \in \partial \omega,
$$

where $\sigma_{0}:=\frac{\mu_{1}+\mu_{0}}{2\left(\mu_{1}-\mu_{0}\right)}$. 
Further, multiplying (78) by $P(x, y)$ and integrating by parts, we obtain

$$
-\int_{\mathbb{R}^{n}} p \operatorname{div}(P) d y=-\int_{\mathbb{R}^{n}} 2 \mu e(u): e(P) d y+\int_{\partial \omega} P(x, y) \cdot f(y) d \sigma_{y}, \quad x \in \mathbb{R}^{n} .
$$

We remark from (37)-(36) that

$$
\operatorname{div}_{y} P(x, y)=-\delta_{x}(y) .
$$

Substituting $\operatorname{div}(P)$ by its expression in (89) we get

$$
p(x)=-\int_{\mathbb{R}^{n}} 2 \mu e(u): e(P) d y+\int_{\partial \omega} P(x, y) f(y) d \sigma_{y}, \quad \forall x \in \mathbb{R}^{n} .
$$

Let $f \in H_{0}^{-1 / 2}(\partial \omega)^{n}$. Since $\mathcal{S}$ is invertible (Lemma B.1), there exists a unique $\varphi \in$ $H_{0}^{\frac{1}{2}}(\partial \omega)$, such that

$$
\left(\sigma_{0} I-\mathcal{K}\right) \varphi(x)=\frac{1}{\left(\mu_{1}-\mu_{0}\right)} \mathcal{S} f(x), \quad \forall x \in \partial \omega .
$$

We then define

$$
\begin{aligned}
& \psi_{1}(x)=\mu_{1} \mathcal{S}^{-1}\left(\frac{1}{2} I+\mathcal{K}\right) \varphi(x), \quad x \in \partial \omega, \\
& \psi_{0}(x)=\mu_{0} \mathcal{S}^{-1}\left(-\frac{1}{2} I+\mathcal{K}\right) \varphi(x), \quad x \in \partial \omega .
\end{aligned}
$$

These functions are in $H_{0}^{-\frac{1}{2}}(\partial \omega)$ and satisfy $\psi_{0}-\psi_{1}=f_{0}$ on $\partial \omega$. Further, we define $(u, p) \in H_{\text {loc }}^{1}\left(\mathbb{R}^{n}\right)^{n} \times L_{\text {loc }}^{2}\left(\mathbb{R}^{n}\right)$ by

$$
u(x):= \begin{cases}\frac{1}{2 \mu_{1}} \mathcal{S} \psi_{1}(x)-\mathcal{D} \varphi(x), & x \in \omega, \\ \mathcal{D} \varphi(x)-\frac{1}{2 \mu_{0}} \mathcal{S} \psi_{0}(x), & x \in \mathbb{R}^{n} \backslash \bar{\omega},\end{cases}
$$

and

$$
p(x):=-\int_{\mathbb{R}^{n}} 2 \mu e(u): e(P) d y+\int_{\partial \omega} P(x, y) f(y) d \sigma_{y} .
$$

These functions are smooth inside $\omega$ and inside $\mathbb{R}^{n} \backslash \bar{\omega}$ and it is easy to check that they satisfy the radiation conditions (79). Using the definition of the Green functions $\boldsymbol{\Gamma}(x, y)$ and $P(x, y)$, using (80), (89) and (90), one checks that $(u, p)$ is a solution of the Stokes system in $\omega$ and in $\mathbb{R}^{n} \backslash \bar{\omega}$ (see also [19], p. 52). Further, the jump conditions (84) show that

$$
\begin{cases}\left.u\right|_{ \pm} & =\varphi \\ \frac{\partial u}{\partial \nu_{1}}-\frac{\partial u}{\partial \nu_{0}} & =f\end{cases}
$$

on $\partial \omega$, which finishes the proof of the proposition.

REMARK B.3. We remark from (37)-(36) that

$$
P=-\nabla \Gamma_{0}, \quad \text { in } \mathbb{R}^{n},
$$

where $\Gamma_{0}$ is the fundamental solution of the Laplacian (given by (47)). Using the last equality and the fact that $\operatorname{div}(u)=0$ in $\mathbb{R}^{n}$, the first term on the right-hand side of the equation (90) can be simplified and written as an integral over $\partial \omega$. 


\section{REFERENCES}

[1] H. Ammari, An introduction to mathematics of emerging biomedical imaging, Math. \& Applications, Vol. 62, Springer-Verlag, Berlin, 2008. MR2440857 (2010j:44002)

[2] H. Ammari, Y. Capdeboscq, H. Kang, H. Lee, G. Milton, and H. Zribi, Progress on the strong Eshelby's Conjecture and extremal structures for the elastic moment tensor, Jour. Math. Pures Appl., 94 (2010), 93-106. MR2653981

[3] H. Ammari, P. Garapon, H. Kang and H. Lee, A method of biological tissues elasticity reconstruction using magnetic resonance elastography measurements, Quart. Appl. Math., 66 (2008) 139-175. MR 2396655 (2009c:35468)

[4] H. Ammari, P. Garapon, H. Kang and H. Lee, Effective viscosity properties of dilute suspensions of arbitrarily shaped particles, to appear in Asymptotic Analysis.

[5] H. Ammari and H. Kang, Reconstruction of Small Inhomogeneities from Boundary Measurements, Lecture Notes in Mathematics, Volume 1846, Springer-Verlag, Berlin (2004). MR2168949 (2006k:35295)

[6] H. Ammari and H. Kang, Polarization and Moment Tensors: with Applications to Inverse Problems and Effective Medium Theory, Applied Mathematical Sciences Series, Vol. 162, Springer-Verlag, New York, 2007. MR2327884 (2009f:35339)

[7] H. Ammari, H. Kang and H. Lee, A boundary integral method for computing elastic moment tensors for ellipses and ellipsoids, J. Comp. Math. 25 (2007) 2-12. MR2292423 (2007k:74011)

[8] H. Ammari, H. Kang, and M. Lim, Effective parameters of elastic composites, Indiana University Mathematics Journal, 55 (2006), 903-922. MR.2244590 (2008g:74086)

[9] H. Ammari, H. Kang, and K. Touibi, Boundary layer techniques for deriving the effective properties of composite materials, Asymptotic Analysis, 41 (2005), 119-140. MR2129229 (2006b:35333)

[10] E. Bonnetier and F. Triki, Asymptotics in the presence of inclusions of small volume for a conduction equation: A case with a non-smooth reference potential, AMS, Contemporary Math. Series, volume 494, pages 95-112, 2009. MR2581768

[11] G.K. Batchelor, The stress system in a suspension of force-free particles, J. Fluid Mech. 41 (1970) 545-570.

[12] Y. Capdeboscq and M. Vogelius, A general representation formula for boundary voltage perturbations caused by internal conductivity inhomogeneities of low volume fraction, Math. Modelling Num. Anal., 37 (2003) 159-173. MR1972656 (2004b:35334)

[13] L. Cattabriga, Su un problema al contorno relativo al sistema di equazioni di Stokes, Rend. Sem. Padova, 31 (1961) 308-340. MR0138894 (25:2334)

[14] D.J. Cedio-Fengya, S. Moskow and M. Vogelius, Identification of conductivity imperfections of small diameter by boundary measurements. Continuous dependence and computational reconstruction, Inverse Problems, 14 (1998) 553-595. MR 1629995 (99d:78011)

[15] M. Do Carmo, Differential geometry of curves and surfaces, Prentice-Hall, 1976. MR0394451 $(52: 15253)$

[16] A. Einstein, Eine neue Bestimmung der Molekuldimensionen, Annalen der Physik, 19 (1906) 289306.

[17] N.E. Jackson, and C. L. Tucker, A model for large deformation of an ellipsoid droplet with interfacial tension, J. Rheol. 47 (2003) 659-682.

[18] H. Kang and G. Milton, Solutions to the Pólya-Szegö conjecture and the weak Eshelby conjecture, Arch. Rational Mech. Anal. 188 (2008), 93-116. MR2379654 (2009j:74046)

[19] O.A. Ladyzhenskaya, The Mathematical Theory of Viscous Incompressible Flow, Second English Edition, Gordon and Breach, New York, 1969. MR0254401 (40:7610)

[20] R. Lipton, On the effective elasticity of a two-dimensional homogenized incompressible elastic composite, Proc. Roy. Soc. Edinburgh Sect. A, 110 (1988) 45-61. MR963839 (91g:73002)

[21] L.P. Liu, Solutions to the Eshelby conjectures, Proc. R. Soc. Lond. Ser. A 464 (2008), 573-594. MR2426985 (2010g:74012)

[22] P.L. Maffettone and M. Minale, Equation of change for ellipsoidal drops in viscous flow, J. NonNewtonian Fluid Mech., 78 (1998) 227-241.

[23] G. Milton, The theory of composites, Cambridge Monographs on Applied and Computational Mathematics, Vol. 6, Cambridge University Press, Cambridge (2002). MR1899805 (2003d:74077)

[24] E. Sánchez-Palencia, Einstein-like approximation for homogenization with small concentration. I. Elliptic problems, Nonlinear Anal., 9 (1985) 1243-1254. MR813656 (87a:35153) 
[25] G.I. Taylor, The viscosity of a fluid containing small drops of another fluid, Proc. R. Soc. Lond. Ser. A 138 (1932) 41-48.

[26] R. Temam, Navier-Stokes equations, theory and numerical analysis, North-Holland, Amsterdam, 1979. MR603444 (82b:35133)

[27] R. C. Tolman, The effect of droplet size on surface tension. Journal of Chemical Physics, 17 (3) (1949) 333-337.

[28] W. Yu and M. Bousmina, Ellipsoidal model for droplet deformation in emulsions, Journal of Rheology, 47 (2003) 1011-1039. 